\title{
A JOURNEY FROM THE HITCHIN SECTION TO THE OPER MODULI
}

\author{
OLIVIA DUMITRESCU
}

To Anthony

\begin{abstract}
This paper provides an introduction to the mathematical notion of quantum curves. We start with a concrete example arising from a graph enumeration problem. We then develop a theory of quantum curves associated with Hitchin spectral curves. A conjecture of Gaiotto, which predicts a new construction of opers from a Hitchin spectral curve, is explained. We give a step-bystep detailed description of the proof of the conjecture for the case of rank 2 Higgs bundles. Finally, we identify the two concepts of quantum curve arising from the topological recursion formalism with the limit oper of Gaiotto's conjecture.
\end{abstract}

\section{Contents}

1. Introduction 2

1.1. Acknowledgments 2

2. Enumeration of ribbon graphs 3

2.1. A combinatorial model for the moduli space of curves $\mathcal{M}_{g, n}$

2.2. The Combinatorics of Catalan recursion 4

2.3. Edge contraction operation and Catalan recursion 4

2.4. Spectral curve for the Catalan recursion 5

2.5. Genesis of Enumerative Geometry: Gromov-Witten invariants of a point 5

2.6. Cut-and-Join equation for orbifold Hurwitz numbers 7

3. A walk into the woods of Higgs bundles and connections

3.1. Moduli spaces of vector bundles 8

3.2. Spectral curve of the Hitchin fibration 9

4. From Higgs bundles to quantum curves 11

4.1. Higgs bundles for Catalan numbers 11

4.2. Higgs bundles and quantum curves 12

5. The metamorphosis of quantum curves into opers 14

5.1. Projective coordinate system

5.2. Hitchin section in rank two

5.3. A family of Deligne's $\hbar$-connections.

6. Hitchin moduli spaces for the Lie group $G=S L_{r}(\mathbb{C}) \quad[19$

6.1. Hitchin section for $S L_{r}(\mathbb{C})$-Higgs bundles (principal $s l_{2}(\mathbb{C})$ ) 21

6.2. On a conjecture of Gaiotto

6.3. Sketch of the proof in rank two

7. The limit oper of Gaiotto's correspondence and the quantum curve

8. Conclusion 27]

\begin{tabular}{ll} 
References & 29 \\
\hline
\end{tabular}

2010 Mathematics Subject Classification. Primary: 58E15, 53C07. Secondary: 14D21, 81T13.

Key words and phrases. Hitchin's equations, moduli space of Higgs bundles, opers, Nonabelian Hodge correspondence, quantum curves.

The author is a member of the Simion Stoilow Institute of Mathematics of the Romanian Academy. 


\section{INTRODUCTION}

Mathematical research is a journey. We start from one place, often a remote place nobody cares. Guided by mysteries one after another, we arrive at a place we have never imagined. We then suddenly realize that many people have come to the same place, starting from totally different origins.

These are the lectures that the author has delivered in the last few years in many places of the world. They are meant to be an introduction to the notion of quantum curves. Yet the honest feeling that the author has now is that these are more a record of how her understanding of quantum curves has evolved. The mathematics of quantum curves itself has been changing over the years. We have started from one place, based on what is known as topological recursion. When we have arrived at the current position, we find ourselves dealing with opers.

The notion of quantum curves was conceived in string theory by Aganagic, Dijkgraaf, Gukov, Hollands, Klemm, Marino, Sulkowski, Vafa, and others [1, 5, 6, 22, We are far from establishing a complete theory at this moment. Yet we hope these lectures give a snapshot of what is understood in the mathematics community now, at least one of the many sides of the story of quantum curves.

This paper is organized as follows. In Section 2, we start from a simple question in enumerative geometry, and obtain the Dijkgraaf-Verlinde-Verlinde formula [7] for intersection numbers of $\psi$ classes on moduli space of stable curves $\overline{\mathcal{M}}_{g, n}$. More precisely, in Section 2.3, we use the edge contraction operations of ribbon graphs to generalize a count of graphs, and establish a recursion of Catalan numbers of arbitrary genera. Then in Section 2.5, we present how the Laplace transform of the recursion of Catalan numbers surprisingly gives the DVV formula for intersection numbers on $\overline{\mathcal{M}}_{g, n}$. By the WKB analysis the recursion relation becomes equivalent to the quantization of the spectral curve of the Catalan numbers. In Section 2.8, we present how the same set of edge contraction operations on ribbon graphs give the cut-and-join equations for orbifold Hurwitz numbers.

We start with presenting an introduction to the geometry of the Hitchin moduli spaces of holomorphic Higgs bundles and connections in Section 4. We then generalize the quantization theorem of Catalan recursion 2.5 replacing the concept of spectral curves of Section 2.4 by the framework of Hitchin spectral curves. More precisely, following [10, 11, 13], we present the quantization results of spectral curves for holomorphic and meromorphic Higgs bundles of rank 2. Here, the algebrogeometric technique presented in Section 4.1 was indispensable in quantizing singular Hitchin spectral curves [11].

In Section 5, using the work of Gunning [23], we propose to identify the two concepts: quantum curves and opers. The new idea of quantization in these notes is based on a recent solution [9] of a conjecture due to the physicist Gaiotto [18, presented in Sections 6 and 7.

1.1. Acknowledgments. The author would like to express her gratitude to the organizers of String-Math 2016 held in Collège de France, Paris, and to the Institute Henri Poincaré, for their hospitality. These lecture notes grew out from the author's paper [9] in collaboration with L. Fredrickson, G. Kydonakis, R. Mazzeo, M. Mulase, and A. Neiztke, that solves a conjecture of Davide Gaiotto [18]. This work was initiated at the AIM workshop, "New perspectives on spectral data for Higgs bundles." The author also thanks the organizers of the workshop, in particular Philip Boalch and Laura Schaposnik, for motivating interest in this problem by posing the question which led to this analysis.

The author is deeply indebted to Motohico Mulase for his generosity in mathematical discussions, enthusiasm, passion and encouragement that stimulated our collaboration throughout the years. This work could not have been produced without his support, for which the author would like to express all her gratitude. 
The research of the author was supported by a grant from the Max-Planck Institute for Mathematics, Bonn. These lectures are based on a collaboration and discussions of the author with Motohico Mulase that took place in 2016 at the Max-Planck Institute for Mathematics, Bonn, and the Institute of Mathematics "Simion Stoilow" in Bucharest. These lectures will be continued in [14, 15].

\section{ENUMERATION OF RIBBON GRAPHS}

2.1. A combinatorial model for the moduli space of curves $\mathcal{M}_{g, n}$. Ribbon graphs are combinatorial objects first used by G. 't Hooft [41] in quantum gauge theory, and later by Kontsevich [27] in random matrix theory as the first approach to Gromov-Witten theory. They appeared independently in the work of Grothendieck [21] and are also known as dessins d'enfants. A ribbon graph as a graph has a cyclic ordering of the set of incident half-edges at each vertex and labeled faces. A ribbon graph embeds into an oriented compact topological surface of type $(g, n)$, where $g$ represents the genus of the surface and $n$ the number of marked points corresponding to the faces of the ribbon graph.

1)

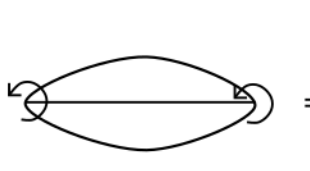

2)

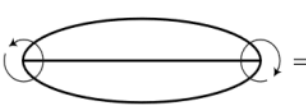

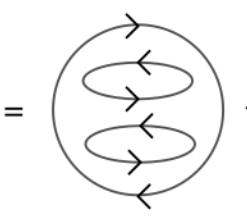

type $(0,3)$
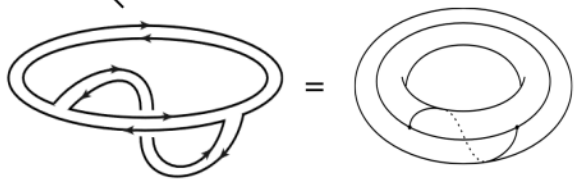

type $(1,1)$

FIG. 1

Decorating ribbon graphs by a positive real number on each edge fixes a topological surface of type $(g, n)$ together with a complex structure on it. Introduce the total space of graphs as an orbifold parametrizing decorated ribbon graphs of a given topological type $(g, n)$ :

$$
\mathcal{R}_{g, n}=\coprod_{\begin{array}{c}
\Gamma \text { boundary labeled } \\
\text { ribbon graph } \\
\text { of type }(g, n) \\
\text { and } e(\Gamma) \text { edges }
\end{array}} \frac{\mathbb{R}_{+}^{e(\Gamma)}}{\operatorname{Aut}(\Gamma)}
$$

The space $\mathcal{R}_{g, n}$ is a smooth orbifold (see [30, Section 3] and [39]). The combinatorial model of moduli space was constructed by Thurston [39, Harer [24, 25], Mumford [33, and Strebel [40] (cf. [30]). There exists an orbifold isomorphism between the total space of graphs of type $(g, n)$ and the product of $\mathbb{R}_{+}^{n}$ and the moduli space $\mathcal{M}_{g, n}$ of smooth algebraic curves of genus $g$ with $n$ marked points:

$$
\mathcal{R}_{g, n} \cong \mathcal{M}_{g, n} \times \mathbb{R}_{+}^{n}
$$

The isomorphism 2.1.1 gives a cell-decompositions of the moduli space $\mathcal{M}_{g, n}$ for each choice of $p \in \mathbb{R}_{+}^{n}$, and generalized Catalan numbers are related to a count of lattice points in each celldecomposition for $p \in \mathbb{Z}_{+}^{n}$. The isomorphism (2.1.1) enables us to use the combinatorial model for the study of topology of $\mathcal{M}_{g, n}$ via ribbon graphs and their geometry. Starting from a count of graphs, or the number of orbi-cells in $\mathcal{R}_{g, n}$, the corresponding enumerative problem on $\mathcal{M}_{g, n}$ surprisingly becomes the intersection numbers of the $\psi$ classes on $\overline{\mathcal{M}}_{g, n}$. 
2.2. The Combinatorics of Catalan recursion. In combinatorics, the Catalan numbers form a sequence of natural numbers that occur in various counting problems for recursively defined objects. They also appear in nature, and have more than twenty alternative definitions. To extend one of these interpretations we define the generalized Catalan number to count a number of graphs on a Riemann surface of genus $g$ with $n$ marked points. We define a cell graph to be a ribbon graph with labeled vertices. We introduce the generalized Catalan numbers, $C_{g, n}\left(\mu_{1}, \ldots, \mu_{n}\right)$, as the number of cell graphs of type $(g, n)$ with an outgoing arrow and degree $\mu_{i}$ at each vertex $i$. In Figure (2(1)), we give an example of a vertex of degree 7 .

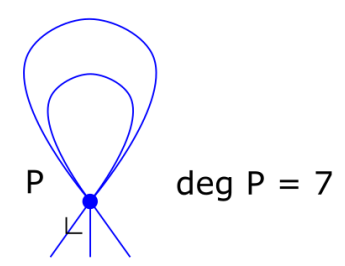

(1)

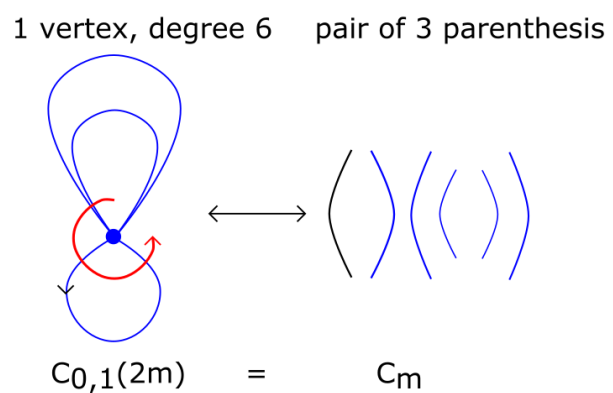

(2)

FIG. 2

Example 2.1. For $(g, n)=(0,1)$, the Catalan numbers $C_{m}$ count graphs on a Riemann sphere with one vertex (so all edges are loops). We recall the classical definition of Catalan numbers counting the number of expressions containing $m$ pairs of parentheses which are correctly matched. It is easy to see that $C_{0,1}(2 m)=C_{m}$. In Figure $(2(2))$ we provide an example explaining why each cell graph corresponds to a pair of parenthesis for $m=3$.

Since for the classical Catalan numbers there is the recursion $C_{m}=\sum_{a+b=m-1} C_{a} \cdot C_{b}$, we expect to find a similar recursion for the generalized Catalan number $C_{g, n}\left(\mu_{1}, \ldots, \mu_{n}\right)$.

\subsection{Edge contraction operation and Catalan recursion.}

Theorem 2.1 (Theorem 3.2, [16], 42]). For $2 g-2+n \geq 0, n \geq 1$, the generalized Catalan numbers satisfy the following recursion

$$
\begin{gathered}
C_{g, n}\left(\mu_{1}, \ldots, \mu_{n}\right)=\sum_{j=2}^{n} \mu_{j} \cdot C_{g, n-1}\left(\mu_{1}+\mu_{j}-2, \mu_{2}, \ldots, \widehat{\mu_{j}}, \ldots, \mu_{n}\right)+ \\
+\sum_{\alpha+\beta=\mu_{1}-2}\left[C_{g-1, n+1}\left(\alpha, \beta, \mu_{2}, \ldots, \mu_{n}\right)+\sum_{\substack{g_{1}+g_{2}=g \\
I \sqcup J=\{2, \ldots, n\}}} C_{g_{1},|I|+1}\left(\alpha, \mu_{I}\right) \cdot C_{g_{2},|J|+1}\left(\beta, \mu_{J}\right)\right] .
\end{gathered}
$$

Proof. Starting from a cell graph with an arrowed edge at vertex 1 we will contract this edge to a point, and we call this an Edge Contraction Operation. We distinguish two cases.

Case 1. The arrowed edge connects vertex 1 of degree $\mu_{1}$ with the vertex $j>1$ of degree $\mu_{j}$. We contract the edge and we join the two vertices 1 and $j$ together as shown in figure below. The resulting graph has one less vertex, but the genus is the same, the degree of the newly created vertex is $\mu_{1}+\mu_{j}-2$; we mark the edge that was immediately counterclockwise of the contracted edge, as indicated in Figure 3(1).

Case 2. The arrowed edge is a loop attached to vertex 1 , then we remove this loop from the cell graph, and separate the vertex into two vertices. The loop classifies all other incident edges into 


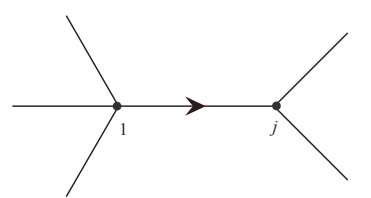

(1)

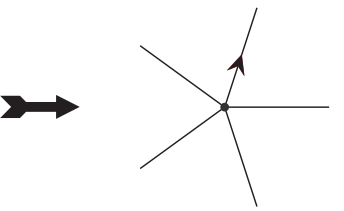

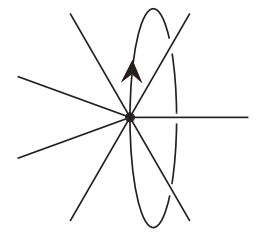

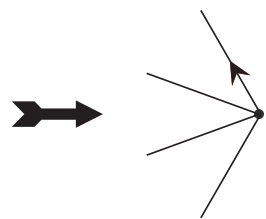

$(2)$

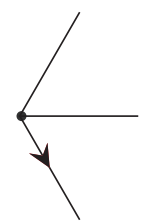

FIG. 3

two groups with $\alpha$ edges in one group, and $\beta$ the rest. Then $\alpha+\beta=\mu_{1}-2$, and we create two vertices of degrees $\alpha$ and $\beta$ as in the Figure $3(2)$,

\subsection{Spectral curve for the Catalan recursion.}

Question 2.2. What is the mirror dual of Catalan numbers?

We will construct the spectral curve of Catalan numbers from the $(g, n)=(0,1)$ unstable geometry. We observe first that the recursion in Theorem 2.1 for the unstable range gives the well-known Catalan recursion of Example 2.1, i.e.,

$$
C_{m}=\sum_{a+b=m-1} C_{a} \cdot C_{b}
$$

We define the generating function of Catalan numbers

$$
z(x):=\sum_{m=0}^{\infty} C_{m} \cdot x^{-(2 m+1)} .
$$

The Catalan recursion (2.4.1) is equivalent to the series $x-z(x)$ being the inverse of the $z(x)$. We thus discover the spectral curve of the Catalan recursion:

$$
z^{2}-z \cdot x+1=0
$$

This is the mirror dual of Catalan numbers.

2.5. Genesis of Enumerative Geometry: Gromov-Witten invariants of a point. By the orbifold isomorphism (2.1.1) we will deduce that the count of graphs is equivalent to an enumerative question on $\overline{\mathcal{M}}_{g, n}$. In the stable range we will consider first the generating function of generalized Catalan numbers, or free energies:

$$
F_{g, n}\left(x_{1}, \ldots, x_{n}\right):=\sum_{\mu_{1}, \ldots, \mu_{n}>0} \frac{C_{g, n}\left(\mu_{1}, \ldots, \mu_{n}\right)}{\mu_{1} \cdots \mu_{n}} \prod_{i=1}^{n} x_{i}^{-\mu_{i}} .
$$

Surprisingly, the generating function $F_{g, n}$ knows $\chi\left(\mathcal{M}_{g, n}\right)$ and intersection numbers of $\overline{\mathcal{M}}_{g, n}$ ! by

We now perform a change of coordinates. For each of the variables $x_{i}$ we introduce a variable $t_{i}$

$$
x_{i}:=2 \cdot \frac{t_{i}^{2}+1}{t_{i}^{2}-1}, \quad z_{i}:=\frac{t_{i}+1}{t_{i}-1} .
$$

With this change of variables $F_{g, n}\left(t_{1}, \ldots, t_{n}\right)$ becomes a Laurent polynomial of degree $3 \cdot(2 n-2+n)$ with beautiful geometric properties

(1) $F_{g, n}(1, \ldots, 1)=(-1)^{n} \chi\left(\mathcal{M}_{g, n}\right)$ 
(2) $F_{g, n}\left(t_{1}, \ldots, t_{n}\right)=F_{g, n}\left(\frac{1}{t_{1}}, \ldots, \frac{1}{t_{n}}\right)$

In Section 4.1 we will answer the following question:

Question 2.3. Why do we have to perform the change of variables (2.5.1) in order to see the topological information encoded by $F_{g, n}$ ?

The leading terms of $F_{g, n}\left(t_{1}, \ldots, t_{n}\right)$ form a homogeneous polynomial of degree $3(2 n-2+n)$

$$
F_{g, n}\left(t_{1}, \ldots, t_{n}\right)^{t o p}=\frac{(-1)^{n}}{2^{2 g-2+n}} \cdot \sum_{\substack{d_{1}+\cdots+d_{n} \\=3 g-3+n}}\left\langle\tau_{d_{1}} \cdots \tau_{d_{n}}\right\rangle \cdot \prod_{i=1}^{n}\left(\left|2 d_{i}-1\right|\right) ! ! \cdot\left(\frac{t_{i}}{2}\right)^{2 d_{i}+1},
$$

where the symbol

$$
\left\langle\tau_{d_{1}} \cdots \tau_{d_{n}}\right\rangle:=\int_{\overline{\mathcal{M}}_{g, n}} c_{1}\left(\mathbb{L}_{1}\right)^{d_{1}} \cdots c_{1}\left(\mathbb{L}_{n}\right)^{d_{n}}
$$

denotes cotangent class intersection numbers on $\overline{\mathcal{M}}_{g, n}$. Furthermore, the Catalan recursion obtained via Edge Contraction Operations in Theorem 2.1 translates into an infinite system of differential equations known as the Dijkgraaf-Verlinde-Verlinde equation [7] of the intersection numbers. This implies the celebrated theorem of Kontsevich, Mirzakhani, Okounkov-Pandharipande and others, on the Witten conjecture.

Theorem 2.4 (Theorem 6.1, [16]). The Witten-Kontsevich intersection numbers satisfy the DVV equation.

Surprisingly, the recursion relations of Theorem 2.4 can be encoded compactly into a single ordinary differential equation. Namely, there exist a differential operator, what we call a quantum curve, that annihilates the generating function of the free energies. More precisely, the first quantization result was proved by Mulase and Sułkowski following a conjecture of Gukov and Sułkowski:

Theorem 2.5 ([31]). Let $\hbar$ be a formal parameter. Then we have

$$
\left(\hbar^{2} \cdot \frac{d^{2}}{d x^{2}}+\hbar \cdot x \cdot \frac{d}{d x}+1\right) \exp \left(\sum_{2 g-2+n \geq-1} \frac{1}{n !} \cdot \hbar^{2 g-2+n} \cdot F_{g, n}(t, \ldots, t)\right)=0 .
$$

Here, the scalar $\hbar$ is a deformation parameter. In Section 5, we will see that mathematically $\hbar$ is an extension class of line bundles on an algebraic curve.

Question 2.6. (1) Why does this complicated function satisfy such a simple differential equation?

(2) Where does this differential equation come from?

Let us replace $z$ by $-\hbar \frac{d}{d x}$ in the the spectral curve equation of the Catalan recursion (2.4.2)

$$
z^{2}-x \cdot z+1=0 .
$$

We obtain precisely the differential operator known as the the quantum curve of the Catalan spectral curve in Theorem 2.5.

The following questions are natural.

$$
\hbar^{2} \cdot \frac{d^{2}}{d x^{2}}+\hbar \cdot x \cdot \frac{d}{d x}+1 .
$$

Question 2.7. $\quad$ (1) From the shape of the above equations, it looks like a canonical quantization of the spectral curve. Is it really the case?

(2) If so, then what is the mathematical framework that explains this surprising phenomenon? 
2.6. Cut-and-Join equation for orbifold Hurwitz numbers. Another example of the use of edge contraction operations on cell graphs gives a surprising enumerative problem of Hurwitz numbers. Let $H_{g, n}^{r}\left(\mu_{1}, \ldots, \mu_{n}\right)$ denote the number of topological types of regular maps from a smooth curve of genus $g$ to $\mathbb{P}^{1}$ with profile $(\overbrace{r, \ldots, r}^{m})$ over $0 \in \mathbb{P}^{1}$, labeled profile $\left(\mu_{1}, \ldots, \mu_{n}\right)$ over $\infty \in \mathbb{P}^{1}$, and simple ramification at any other ramification points, weighted with automorphisms of such maps. These numbers are referred to as orbifold Hurwitz numbers. For $r=1$, they count simple Hurwitz numbers. In [12] we generalized the notion of branching graph of Okounkov and Pandharipande [36] $(r=1)$ to $r$-Hurwitz graphs (arbitrary $r$ ), via combinatorics. An $r$-Hurwitz graphs is a cell graph with a collection of dots associated to each vertex ([Definition 6.1, [12]]). Counting the number of $r$-Hurwitz graphs via edge contraction operations we recover the Cut-andJoin equation for orbifold Hurwitz numbers. Denoting $\mathcal{H}_{g, n}^{r}\left(\mu_{1} \ldots, \mu_{n}\right)=\mu_{1} \ldots \mu_{n} H_{g, n}^{r}\left(\mu_{1} \ldots, \mu_{n}\right)$, we obtain

Theorem 2.8 (Theorem 6.6, [12], Cut-and-Join equation for orbifold Hurwitz numbers). The number of arrowed Hurwitz graphs satisfy the following edge-contraction formula.

$$
\begin{aligned}
& \left(2 g-2+\frac{d}{r}+n\right) \mathcal{H}_{g, n}^{r}\left(\mu_{1} \ldots, \mu_{n}\right) \\
& =\sum_{i<j} \mu_{i} \mu_{j} \mathcal{H}_{g, n-1}^{r}\left(\mu_{1}, \ldots, \mu_{i-1}, \mu_{i}+\mu_{j}, \mu_{i+1}, \ldots, \widehat{\mu_{j}}, \ldots, \mu_{n}\right) \\
& +\frac{1}{2} \sum_{i=1}^{n} \mu_{i} \sum_{\substack{\alpha+\beta=\mu_{i} \\
\alpha, \beta \geq 1}}\left[\mathcal{H}_{g-1, n+1}^{r}\left(\alpha, \beta, \mu_{1}, \ldots, \widehat{\mu_{i}}, \ldots, \mu_{n}\right)\right. \\
& \left.+\sum_{\substack{g_{1}+g_{2}=g \\
I \sqcup J=\{1, \ldots, \hat{i}, \ldots, n\}}} \mathcal{H}_{g_{1},|I|+1}^{r}\left(\alpha, \mu_{I}\right) \mathcal{H}_{g_{2},|J|+1}^{r}\left(\beta, \mu_{J}\right)\right] .
\end{aligned}
$$

Here, ${ }^{-}$indicates the omission of the index, and $\mu_{I}=\left(\mu_{i}\right)_{i \in I}$ for any subset $I \subset\{1,2, \ldots, n\}$.

The restriction to the $(0,1)$ unstable cases of Theorem 2.8 recovers the spectral curve of the orbifold Hurwitz numbers, that is known as the r-Lambert curve. The computations are similar to the ones explained in Section 2.2, leading to the mirror curve of Hurwitz numbers

$$
x^{r}=y e^{-r y} \text {. }
$$

Edge contraction operations are graphical manifestation of a Frobenius algebra structure and it was shown in [Corollary 4.8, [12])] that they give alternative axiomatic definition of 2 dimensional topological quantum field theory (2d TQFT). We further emphasize the importance of these operations by relating the $2 \mathrm{~d}$ TQFT formula of [Corollary 4.8, [12])] with the count of points of a character variety for a finite group and Hodge-Deligne polynomial of a character variety in [14, 15].

While Catalan numbers have an algebraic spectral curve, Hurwitz numbers have an analytic spectral curve. Later on we will focus on rank 2 Higgs bundles, whose spectral curves are algebraic. In Section 4.1, we will encounter with the familiar Catalan example.

\section{A walk into the woods of Higgs Bundles And CONnections}

Let $C$ be a smooth projective curve, and $K_{C}$ the canonical bundle of $C$ whose sections are holomorphic 1-forms. 
3.1. Moduli spaces of vector bundles. We recall the somewhat anachronistic definition of a holomorphic vector bundle $E$ over $C$. For an open cover of affine sets $C=\cup_{\alpha} U_{\alpha}$, we denote by $f_{\alpha \beta}: U_{\alpha} \cap U_{\beta} \rightarrow G L_{r}(\mathbb{C})$ the holomorphic transition functions that satisfy the 1-cocyle condition $f_{\alpha \beta}=f_{\alpha \gamma} \cdot f_{\gamma \beta}$ on $U_{\alpha} \cap U_{\beta} \cap U_{\gamma}$. Two points $\left(x_{\alpha}, \zeta_{\alpha}\left(x_{\alpha}\right)\right) \in U_{\alpha} \times \mathbb{C}^{r}$ and $\left(x_{\beta}, \zeta_{\beta}\left(x_{\beta}\right)\right) \in U_{\beta} \times \mathbb{C}^{r}$ are glued if $\zeta_{\alpha}(x)=f_{\alpha \beta}(x) \cdot \zeta_{\beta}(x)$ for $x \in U_{\alpha} \cap U_{\beta}$. Two transition functions $f_{\alpha \beta}$ and $f_{\alpha \beta}^{\prime}$ subordinating the same open covering $U_{\alpha}$ define the isomorphic vector bundle if and only if there exists a family $u_{\alpha}: U_{\alpha} \rightarrow G L_{r}(\mathbb{C})$ of holomorphic maps, called gauge transformations, satisfying

$$
f_{\alpha \beta}^{\prime}=u_{\alpha} \cdot f_{\alpha \beta} \cdot u_{\beta} .
$$

A global holomorphic section of the vector bundle $E, s \in H^{0}(C, E)$, is given by a collection of holomorphic maps $s_{\alpha}: U_{\alpha} \rightarrow \mathbb{C}^{r}$ compatible with the transition functions: $s_{\alpha}(x)=f_{\alpha \beta}(x) \cdot s_{\beta}(x)$ for $x \in U_{\alpha} \cap U_{\beta}$.

The degree of a vector bundle is the first Chern class, $\operatorname{deg}(E):=c_{1}\left(\Lambda^{r}(E)\right)$. Over a compact connected Riemann surface $C$, topologically vector bundles are completely classified by the discrete invariants, rank and degree. However, introducing a holomorphic structure, classification results of vector bundles on a smooth, irreducible, complex projective curve become more elaborate. Define the slope of $E$ to be the rational number $\mu(E)=\operatorname{deg}(E) / \operatorname{rank}(E)$ - this is a topological quantity with important implications on holomorphic structures. A holomorphic vector bundle $E$ is called stable (resp. semi-stable) if for any non-trivial holomorphic subbundle $F, \mu(F)<\mu(E)$ (resp. $\mu(F) \leq \mu(E))$ holds. There are complete classification results for holomorphic vector bundles for rational and elliptic curves, due to Grothendieck for the case of the Riemann sphere [20], and Atiyah for the case of elliptic curves [2. However, for genus higher than one there are no such classification results available, therefore such question is replaced by the construction of moduli space of stable holomorphic vector bundles of rank $r$ and degree $e$, denoted by $\mathcal{U}_{C}(r, e)$, whose geometry has been intensely studied. Over a smooth projective curve $C$ of genus $g>1$, the moduli space $\mathcal{U}_{C}(r, e)$ is a quasi-projective complex variety of dimension $r^{2}(g-1)+1$ (Narasimhan-Seshadri [35], Seshadri [37, see also Atiyah-Bott [3] and Mumford-Fogarty-Kirwan [34] for more information on the moduli theory of stable vector bundles over Riemann surfaces).

By reducing the Yang-Mills self-duality equations from dimension 4 to dimension 2 on a compact Riemann surface, Hitchin introduced in [26] the moduli space of solutions to Hitchin's equations, having a $\mathbb{C P}^{1}$ of complex structures, parametrized by $\zeta \in \mathbb{C P}^{1}$. If $\zeta$ is zero, then this space is identified with the Dolbeault moduli space $\mathcal{M}_{\text {Dol }}$ of holomorphic stable Higgs bundles consisting of $(E, \phi)$, where $E$ is a vector bundle of rank $r$ and fixed degree $e$, and $\phi \in H^{0}\left(C, \operatorname{End}(E) \otimes K_{C}\right)$ is a Higgs field. If $r$ and $e$ are coprime, then $\mathcal{M}_{\text {Dol }}$ becomes quasi-projective variety. If $\zeta$ is non-zero and $e=0$, then the the moduli space of solutions to Hitchin's equations can be identified, as a complex analytic variety, with the de Rham moduli space $\mathcal{M}_{\mathrm{deR}}$ consisting of irreducible flat connections $\nabla$ in holomorphic vector bundles $V$ of rank $r$ and degree 0 .

The cotangent bundle $T^{*} \mathcal{U}_{C}(r, e)$ is an open dense subset of $\mathcal{M}_{\text {Dol }}$ whose complement has codimension 2 or higher. Therefore, $\operatorname{dim} \mathcal{M}_{\text {Dol }}=\operatorname{dim} T^{*} \mathcal{U}_{C}(r, e)=2 \operatorname{dim} \mathcal{U}_{C}(r, e)=2 \cdot r^{2} \cdot(g-1)+2$, and it acquires a holomorphic symplectic structure.

These two moduli spaces, $\mathcal{M}_{\text {Dol }}$ and $\mathcal{M}_{\text {deR }}$, will play a key role in our discussion.

Definition 3.1. Let $C$ be a smooth projective curve of genus at least two, $E$ and $V$ two holomorphic rank r vector bundles on $C$ and $d$ the exterior differential on $C$.

(1) A holomorphic Higgs bundle is a pair $(E, \phi)$, where $E$ is a holomorphic vector bundle and $\phi: E \rightarrow E \otimes K_{C}$ is a $\mathcal{O}_{\mathcal{C}}$-module homomorphism, i.e., $\phi(f \cdot s)=f \cdot \phi(s), \forall f \in \mathcal{O}_{\mathcal{C}}, s \in E$.

(2) A stable (resp. semi-stable) Higgs bundle is a Higgs pair $(E, \phi)$ such that for any $\phi$-invariant sub-bundle $F$ of $E, \phi: F \rightarrow F \otimes K_{C}, \mu(F)<\mu(E)$ (resp. $\mu(F) \leq \mu(E)$ ) holds. 
(3) A holomorphic connection is a $\mathbb{C}$-linear homomorphism $\nabla: V \rightarrow V \otimes K_{C}$ of a holomorphic vector bundle $V$ such that $\nabla(f \cdot s)=d f \otimes s+f \cdot \nabla(s), \forall f \in \mathcal{O}_{\mathcal{C}}$ and holomorphic sections $s \in V$. A differentiable connection is defined in the same way, replacing $f$ by a differentiable function on $C$ and $s \in V$ by a differentiable section of $V$.

(4) An irreducible connection is a connection $\nabla$ in $V$ for which no sub-bundle of $V$ is $\nabla$-invariant.

(5) A hermitian metric on the complex vector bundle $E$ is a positive definite Hermitian form $h$ on each fiber $E_{p}, p \in C$. It is a smooth section of $\Gamma\left(E \otimes \bar{E}^{*}\right)$ such that for all $\eta, \zeta \in E_{p}$,

$$
\langle\eta, \zeta\rangle:=h_{p}(\eta, \bar{\zeta})=h_{p}(\zeta, \bar{\eta}) \text { and } h_{p}(\zeta, \bar{\zeta})>0 \text {. }
$$

(6) Let $h$ be a hermitian metric in a vector bundle $V$, and $\langle\cdot, \cdot\rangle$ the hermitian inner product. An $h$-unitary connection on a vector bundle $V$ is a differentiable connection $\nabla$ such that for any differentiable sections $s$ and $t$ of $E,\langle s, t\rangle=\langle\nabla(s), \nabla(t)\rangle$.

Remark 3.2. If $\nabla_{1}$ and $\nabla_{2}$ are holomorphic connections in a holomorphic vector bundle $V$, then the difference $\nabla_{1}-\nabla_{2}$ is an $\mathcal{O}_{C}$-module homomorphism. Therefore, $\left(V, \nabla_{1}-\nabla_{2}\right)$ is a holomorphic Higgs pair. This proves that the Dolbeault and the de Rham moduli spaces, $\mathcal{M}_{\text {Dol }}$ and $\mathcal{M}_{\text {deR }}$, have the same dimensions.

3.2. Spectral curve of the Hitchin fibration. The Higgs field $\phi: E \rightarrow E \otimes K_{C}$ induces a map $\wedge^{i} \phi: \wedge^{i} E \rightarrow \wedge^{i} E \otimes K_{C}^{i}$ for every $i \geq 0$, locally given by the alternative sum of the $i$-th minors of $\phi$, with trace $\operatorname{tr}\left(\wedge^{i} \phi\right) \in H^{0}\left(C, K_{C}^{i}\right)$. If $i=r$ then $\wedge^{r} \phi=\operatorname{tr}\left(\wedge^{r} \phi\right)=\operatorname{det} \phi \in$ $H^{0}\left(C, \operatorname{End}\left(\wedge^{r} E\right) \otimes K_{C}^{r}\right)$. This defines a holomorphic map, called the Hitchin map $H$, which induces an algebraically completely integrable Hamiltonian system

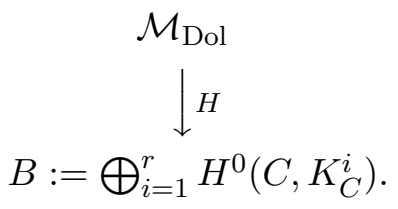

For a Higgs pair $(E, \phi)$ let $s$ denote the spectral data

$$
s:=\left((-1)^{i} \operatorname{tr}\left(\wedge^{i} \phi\right)\right)_{i=1, \ldots, r} \in B \cong \mathbb{C}^{r^{2}(g-1)+1} .
$$

The Hitchin map $H$ sends a Higgs bundle to its spectral data

$$
(E, \phi) \stackrel{H}{\rightarrow} s .
$$

Obviously, the fiber of $H$ over zero contains all Higgs bundles of the form $(E, \phi=0)$, where $E$ is a stable vector bundle, so $\mathcal{U}_{C}(r, e) \subset H^{-1}(0)$.

The total space of the canonical bundle $K_{C}$ is the cotangent bundle $T^{*} C$. There is a tautological 1-form $\eta \in H^{0}\left(T^{*} C, \pi^{*} K_{C}\right)$ on the symplectic variety $T^{*} C$ defined by

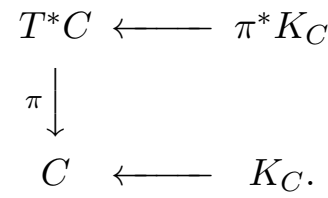

Locally $\eta$ is defined by $y d x$ for $x \in C$ and $y$ the fiber coordinate, while $-d \eta$ is the natural holomorphic symplectic form on $T^{*} C$.

The characteristic polynomial of a Higgs bundle $(E, \phi)$ defines the spectral curve denoted by $\Sigma_{s}$ in $T^{*} C$ as the divisor of the zero of the following global section

$$
\operatorname{det}\left(\eta \cdot I_{r}-\pi^{*} \phi\right):=\sum_{i=0}^{r}(-1)^{i} \operatorname{tr}\left(\wedge^{i} \phi\right) \otimes \eta^{\otimes(r-i)} \in H^{0}\left(T^{*} C, \pi^{*} K_{C}^{r}\right) .
$$


The coefficients of the defining equation of $\Sigma_{s}$ are given by the spectral data $s$ of the Higgs bundle $(E, \phi)$. Observe that $\eta$ induces a 1 -form on the spectral curve $\Sigma_{s}$ by pulling back via the inclusion map $i$ of $\Sigma_{s}$ in $T^{*} C$.

Remark 3.3. General Properties of $\Sigma_{s}$.

(1) $\Sigma_{s}$ is non-singular for generic $s$.

(2) $\Sigma_{s}$ is a curve inside the cotangent bundle $T^{*} C$ of genus

$$
g(\Sigma)=r^{2}(g-1)+1 .
$$

(3) The fiber over a generic point is the Jacobian:

$$
H^{-1}(s)=\operatorname{Jac}\left(\Sigma_{s}\right)
$$

(4) There is a degree $r$ cover

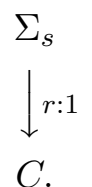

From now on we will consider the Hitchin theory for the Lie group $G=S L_{r}(\mathbb{C})$. In other words, $\mathcal{M}_{\text {Dol }}$ denotes the moduli space of holomorphic Higgs bundles $(E, \phi)$ with $\operatorname{tr}(\phi)=0$ such that $E$ has the fixed trivial determinant. The fiber of the Hitchin map $H^{-1}(s)$ at a generic point

$$
s \in B:=\bigoplus_{i=2}^{r} H^{0}\left(C, K_{C}^{i}\right)
$$

becomes the Prym variety

$$
H^{-1}(s)=\operatorname{Prym}\left(\Sigma_{s} \rightarrow C\right):=\operatorname{Ker}(N m)
$$

of the spectral covering $\pi: \Sigma_{s} \longrightarrow C$ with the spectral data $s$, i.e., the kernel of the norm map

$$
N m: \operatorname{Jac}\left(\Sigma_{s}\right) \ni \sum_{p \in \Sigma_{s}} m_{p} \cdot p \longmapsto \sum_{p \in \Sigma_{s}} m_{p} \cdot \pi(p) \in \operatorname{Jac}(C) .
$$

The moduli space $\mathcal{M}_{\text {Dol }}$ is a generically Abelian fibration over $B$.

3.2.1. Rank 2 simplification. We will focus next on rank 2 and degree 0.

(1) The $S L_{2}(\mathbb{C})$-Higgs bundle $(E, \phi)$, has $\operatorname{tr}(\phi)=0$ and trivial determinant $\operatorname{det}(E)=\wedge^{2} E=\mathcal{O}_{C}$.

(2) The Hitchin map

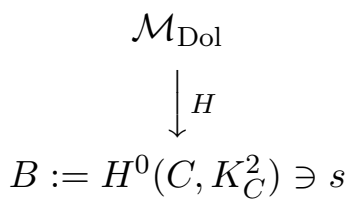

sends $(E, \phi) \stackrel{H}{\rightarrow} \operatorname{det}(\phi)=s$.

Example 3.1 (rank two $S L_{2}(\mathbb{C})$ stable Higgs bundles). Choose a spin structure on a curve $C$ of genus $g \geq 2$, i.e., a choice of the line bundle $K_{C}^{\frac{1}{2}}, 5.1 .2$. For any quadratic differential $q \in$ $H^{0}\left(C, K_{C}^{2}\right)$ on $C,\left(K_{C}^{\frac{1}{2}} \oplus K_{C}^{-\frac{1}{2}},\left(\begin{array}{ll}0 & q \\ 1 & 0\end{array}\right)\right)$ is a stable Higgs bundle on $C$. This example will play a key role in our later analysis (see Definition (5.2)).

Another example is on the trivial vector bundle. For any non-zero holomorphic 1-form $p \in$ $H^{0}\left(C, K_{C}\right),\left(\mathcal{O}_{C} \oplus \mathcal{O}_{C},\left(\begin{array}{ll}0 & p \\ p & 0\end{array}\right)\right)$ is again a stable Higgs bundle. 
Remark 3.4. Let $\left\{f_{\alpha \beta}\right\}$ be a transition function for $E$.

(1) Locally on $C,\left.\nabla\right|_{U}=d+\left.A\right|_{U}$, where $A: E \rightarrow E \otimes K_{C}$.

(2) Any Higgs field is compatible with the transition functions of $E$. Indeed, if $\phi_{\alpha}=\left.\phi\right|_{U_{\alpha}}$, then

$$
\phi_{\alpha}=f_{\alpha \beta} \cdot \phi_{\beta} \cdot f_{\alpha \beta}^{-1} \text {. }
$$

(3) If $s \in E$ is a holomorphic section, then $s_{\alpha}(x)=f_{\alpha \beta}(x) \cdot s_{\beta}(x)$ for $x \in U_{\alpha} \cap U_{\beta}$. We note that derivatives of sections do not make sense as sections: $d s_{\alpha}(x) \neq f_{\alpha \beta}(x) \cdot d s_{\beta}(x)$. Nevertheless, if $A_{\alpha}=\left.A\right|_{U_{\alpha}}$, then $\left(d+A_{\alpha}\right) \cdot s_{\alpha}=f_{\alpha \beta} \cdot\left[\left(d+A_{\beta}\right) \cdot s_{\beta}\right]$ on $U_{\alpha} \cap U_{\beta}$. Therefore, for any connection $\nabla=\left\{d+A_{\alpha}\right\}_{\alpha}$ on $E$, the Gauge transformation holds

$$
A_{\alpha}=f_{\alpha \beta} \cdot A_{\beta} \cdot f_{\alpha \beta}^{-1}-f_{\alpha \beta}^{-1} \cdot d f_{\alpha \beta} .
$$

Since $f_{\alpha \beta} \cdot f_{\alpha \beta}^{-1}=1$, by applying the exterior differential $d$, it is obvious that 3.2 .4 is equivalent to $A_{\alpha}=f_{\alpha \beta} \cdot A_{\beta} \cdot f_{\alpha \beta}^{-1}+f_{\alpha \beta} \cdot d f_{\alpha \beta}^{-1}$, or simply $d f_{\alpha \beta}=f_{\alpha \beta} \cdot A_{\beta}-A_{\alpha} \cdot f_{\alpha \beta}$.

Locally, $\phi$ and $A$ are both $r$ by $r$ matrices of 1-forms, but satisfying different rules with respect to the transition function of the vector bundle. We emphasize here that given a Higgs bundle $(E, \phi)$ in $\mathcal{M}_{\text {Dol }}$, it is not obvious how to obtain a connection $(V, \nabla)$ in $\mathcal{M}_{\mathrm{deR}}$. The goal of these lecture notes is to reveal the holomorphic path that a Higgs bundle on a Hitchin section travels to become a connection, called an oper, is the goal of these lecture notes.

\section{From Higgs bundles to quantum Curves}

4.1. Higgs bundles for Catalan numbers. We wish to consider the curve $z^{2}-z \cdot x+1=0$ of 2.4.1) as a local expression of a singular spectral curve (divisor) inside the Hirzebruch surface $\mathbb{F}_{2}$ associated with a meromorphic Higgs bundle.

Example 4.1. The Spectral curve of Catalan numbers as the spectral curve of a Higgs bundle.

(1) The curve is $C=\mathbb{P}^{1}$, and the vector bundle $E=K_{C}^{\frac{1}{2}} \oplus K_{C}^{-\frac{1}{2}}=\mathcal{O}_{\mathbb{P}^{1}}(-1) \oplus \mathcal{O}_{\mathbb{P}^{1}}(1)$.

(2) The meromorphic Higgs field $\phi: E \rightarrow E \otimes K_{C}(*)$ is given by

$$
\phi=\left(\begin{array}{cc}
0 & -(d x)^{2} \\
1 & x \cdot d x
\end{array}\right)
$$

on the affine line $\mathbb{A}^{1} \subset \mathbb{P}^{1}$.

(3) The spectral curve of a Higgs bundle, denoted by

$$
\Sigma \subset \overline{T^{*} \mathbb{P}^{1}}=\mathbb{F}_{2}:=\mathbb{P}\left(\mathcal{O}_{\mathbb{P}^{1}}(-2) \oplus \mathcal{O}_{\mathbb{P}^{1}}\right),
$$

is given by the characteristic polynomial of the Higgs field $\phi$

$$
\operatorname{det}\left(\eta \cdot I_{2}-\phi\right)=\operatorname{det}\left(z \cdot d x \cdot I_{2}-\phi\right)=\left(z^{2}-x \cdot z+1\right) \cdot(d x)^{2}=0
$$

in $T^{*} \mathbb{A}^{1}$.

- We further consider a resolution of singularity of curve $\Sigma$ by blowing up $\mathbb{F}_{2}$.

- $\Sigma$ is smooth in $T^{*} \mathbb{A}^{1}$ near $(0,0)$ (Figure 4(1)), and has a double point in $\mathbb{F}_{2}$ at $(\infty, \infty)$ (Figure 4(2)].

- Blow up the surface $\mathbb{F}_{2}$ and denote by $\widetilde{\Sigma}$ the strict transform of $\Sigma$. Introduce a new local parameter $w_{1}$ such that $w=w_{1} \cdot u$. The strict transform $\widetilde{\Sigma}$ becomes a conic

$$
\widetilde{\Sigma}: u^{2}+\left(w_{1}-\frac{1}{2}\right)^{2}=\frac{1}{4} \text {. }
$$




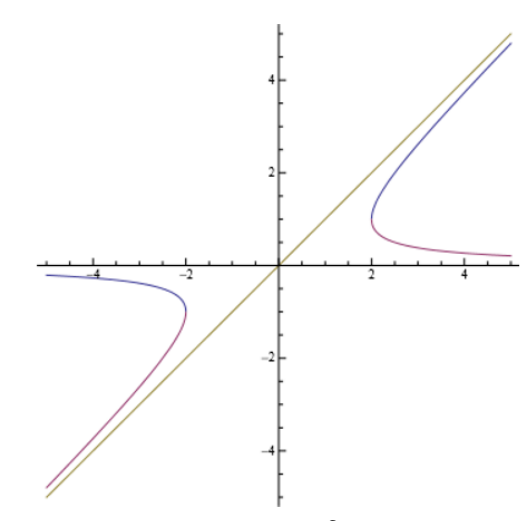

(1): $\Sigma$ around $(0,0): z^{2}-x \cdot z+1=0$

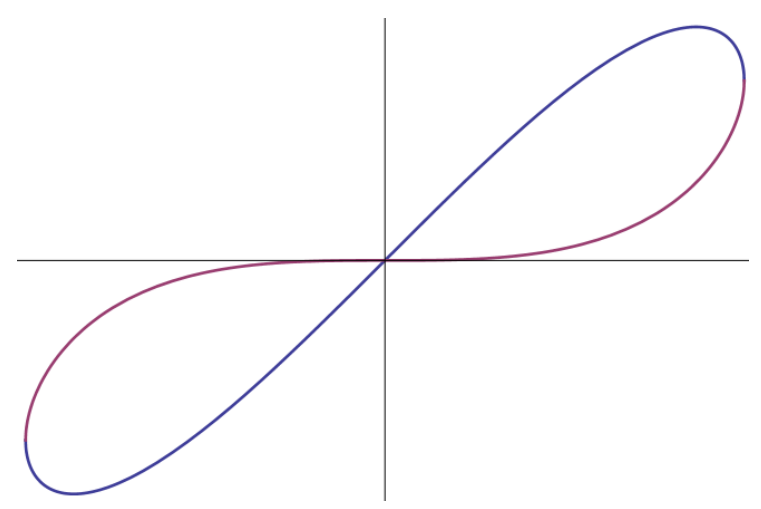

(2): $\Sigma$ at $(\infty, \infty): u^{4}-u \cdot w+w^{2}=0$

FiG. 4

- $\widetilde{\Sigma}$ is a rational curve, and $t$ of 2.5 .1$)$ is the normalization coordinate that parametrizes the affine part of the spectral curve by

$$
\begin{aligned}
u & =\frac{t^{2}-1}{2 \cdot\left(t^{2}+1\right)} \\
w_{1} & =\frac{1}{2}-\frac{t}{t^{2}+1} .
\end{aligned}
$$

- Denote by $F$ the class of a fiber of $\mathbb{F}_{2} \rightarrow \mathbb{P}^{1}$, by $B$ the negative section, i.e., the zero section of $T^{*} \mathbb{P}^{1}$, and by $E$ the exceptional divisor created on $\mathbb{F}_{2}$ after the blow-up at the double point of $\Sigma$ at $(\infty, \infty)$. Then the proper transform $\widetilde{\Sigma}$ on the blown-up of $\mathbb{F}_{2}$ is written as the divisor

$$
\widetilde{\Sigma}=4 F+2 B-2 E .
$$

4.2. Higgs bundles and quantum curves. We are now ready to generalize Theorem 2.5 and results in Section 4.1 to any meromorphic Higgs bundle $(E, \phi)$ of rank two.

In [10] we have established a new connection between the Hitchin theory/Higgs bundles and topological recursion/quantum curve theory. These are two apparently different broad theories that share the notion of spectral curves. To establish the notations, let $C$ be a smooth projective curve of arbitrary genus, and $K_{C}$ the canonical bundle. We denote by $E$ a holomorphic rank two vector bundle on $C$, and by $\phi: E \rightarrow E \otimes K_{C}(*)$ a Higgs field.

- In [10] we considered a holomorphic Higgs pair $(E, \phi)$. Hitchin constructed the spectral curve $\Sigma$ of $\phi$ by the characteristic polynomial of $\phi(3.2 .2), \Sigma \hookrightarrow T^{*} C$.

- In [11] we considered a meromorphic Higgs pair. We construct the spectral curve $\Sigma$ as the zero divisor of the characteristic polynomial of $\phi$ inside the compactified cotangent bundle of $C$ that is a ruled surface over $C$ :

$$
\Sigma:=\left(\operatorname{det}\left(\eta \cdot I_{r}-\pi^{*} \phi\right)\right)_{0} \hookrightarrow \overline{T^{*} C},
$$

where $\eta \in H^{0}\left(T^{*} C, \pi^{*} K_{C}\right)$ is the tautological 1-form on $T^{*} C$ extended as a meromorphic 1-form on the compactification $\overline{T^{*} C}$. We consider a resolution of singularities of $\Sigma$ by 
blowing up the ruled surface $\overline{T^{*} C}$ over $C$, along the base locus of $\Sigma$.

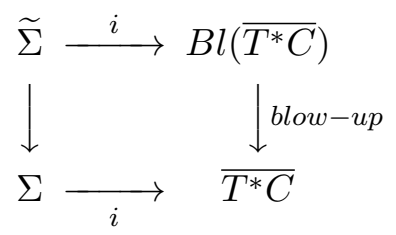

In [10], [11] (see also [13]), we extended the framework of topological recursion [17] to singular Hitchin spectral curves, utilizing the birational geometry of ruled surfaces. As a consequence, this extension has led to the discovery of the relation between Hitchin spectral curves and GromovWitten invariants in few examples (as the one in Section 2.1 and Section 4.1). More precisely, the novelty of this approach is the discovery of the PDE differential recursions of free energies $F_{g, n}$ in [Definition 6.6, [11]] (as well as [Equation 6.5, [10]]) that implies the WKB analysis of the quantization Theorem 4.1. Moreover, the PDE differential recursions of free energies $F_{g, n}$ also implies the well-known integral topological recursion of Eynard-Orantin for a spectral curve of genus 0 . The PDE recursion relates the Hitchin spectral curve with enumerative geometry.

Theorem 4.1 (Quantization Theorem [10, [11]). For a rank 2 Higgs bundle and $x \in C$, we construct locally a second order differential operator $P(x, \hbar \cdot d / d x)$ whose semi-classical limit recovers the spectral curve $\Sigma$. We also construct a solution $\psi(x, \hbar)$ of equation $P(x, \hbar \cdot d / d x) \psi(x, \hbar)=0$ in terms of principal specialization of the PDE recursion.

The enumerative geometry example of the Catalan numbers emphasized by equation $(2.4 .2)$ is locally encaptured in the framework of Hitchin systems by Example in the Section 4.1. Following this approach, assume that the spectral curve of the Higgs bundle has the local expression

$$
y^{2}-\operatorname{tr} \phi(x) \cdot y+\operatorname{det} \phi(x)=0 .
$$

The quantum curve associated to this spectral curve is a Rees $D$-module, locally given by the second order differential operator obtained by replacing the $y$ variable by $\hbar \frac{d}{d x}$ (as in Theorem 2.5)

$$
P(x, \hbar \cdot d / d x)=\left(\hbar \cdot \frac{d}{d x}\right)^{2}-\operatorname{tr} \phi(x) \cdot \hbar \cdot \frac{d}{d x}+\operatorname{det} \phi(x) .
$$

The generating function of free energies is

$$
\psi(x, \hbar)=\exp \left(\sum_{2 g-2+n \geq-1} \frac{1}{n !} \cdot \hbar^{2 g-2+n} \cdot F_{g, n}(x, \ldots, x)\right)=0,
$$

where $F_{g, n}\left(x_{1}, \ldots, x_{n}\right)$ are the free energies defined by the PDE recursion of [Definition 6.6, [11]]. If the spectral curve $\Sigma$ is a singular curve, then the differential operator $P(x, \hbar \cdot d / d x)$ has irregular singularities and $\psi$ has essential singularities. The asymptotic expansion of $\psi$ (see e.g. [Definition 1.1, [13]]) as in the Catalan example (2.4.2) around its singularity has coefficients that encode information of Gromov-Witten invariants (Section 2.5, see also the Airy example of [Section 1, [13]). 


\section{The METAMORPhosis OF QUANTUM CURVES INTO OPERS}

From now on we will focus on holomorphic Higgs bundles $(E, \phi)$ on a Riemann surface $C$ of genus at least two.

5.1. Projective coordinate system. We recall that a universal covering is a covering space that is simply connected. By Riemann uniformization theorem, every simply connected Riemann surface is biholomorphic to $\mathbb{P}^{1}, \mathbb{C}$, or to the upper half-plane $\mathbb{H}:=\{z \in \mathbb{C} \mid \operatorname{Im}(z)>0\}$ with a global coordinate $z$. Therefore for a Riemann surface of genus at least two, the universal covering is the upper half-plane.

Notice that the global coordinate on $\mathbb{H}$ induces, by the quotient map $\pi: \mathbb{H} \rightarrow C$, a particular coordinate system on the Riemann surface $C$. Indeed, there is a faithful representation

$$
\rho: \pi_{1}(C) \longrightarrow S L(2, \mathbb{R})
$$

such that $C \cong \mathbb{H} / \rho\left(\pi_{1}(C)\right)$, where $S L(2, \mathbb{R})$ acts on $\mathbb{H}$ through the projection

$$
0 \longrightarrow \mathbb{Z} / 2 \mathbb{Z} \longrightarrow S L(2, \mathbb{R}) \longrightarrow P S L(2, \mathbb{R})=\operatorname{Aut}(\mathbb{H}) \longrightarrow 0 .
$$

We can give a particular coordinate system on $C$ using the universal covering map $\pi: \mathbb{H} \longrightarrow C$. Let

$$
C=\bigcup_{\alpha} U_{\alpha}
$$

be an open finite cover of $C$. For each coordinate neighborhood $U_{\alpha}$, choose a contractible open subset $\widetilde{U}_{\alpha} \subset \mathbb{H}$ for which the map

$$
\pi: \widetilde{U}_{\alpha} \stackrel{\sim}{\longrightarrow} U_{\alpha} \subset C
$$

is a biholomorphic map. Let us denote by $z_{\alpha}$ the local coordinate defined on $U_{\alpha}$ that corresponds to the global coordinate $z$ restricted on $\widetilde{U}_{\alpha}$. Then on each $U_{\alpha} \cap U_{\beta}$, we have a Möbius coordinate transformation

$$
z_{\alpha}=\frac{a_{\alpha \beta} \cdot z_{\beta}+b_{\alpha \beta}}{c_{\alpha \beta} \cdot z_{\beta}+d_{\alpha \beta}}, \quad\left[\begin{array}{cc}
a_{\alpha \beta} & b_{\alpha \beta} \\
c_{\alpha \beta} & d_{\alpha \beta}
\end{array}\right] \in S L(2, \mathbb{R}) .
$$

In what follows, we choose and fix a Möbius coordinate system on $C$.

Since

$$
d z_{\alpha}=\frac{1}{\left(c_{\alpha \beta} \cdot z_{\beta}+d_{\alpha \beta}\right)^{2}} \cdot d z_{\beta},
$$

the transition function for the canonical line bundle $K_{C}$ of $C$ is given by the cocycle

$$
\left\{\xi_{\alpha \beta}=\frac{d z_{\beta}}{d z_{\alpha}}=\left(c_{\alpha \beta} \cdot z_{\beta}+d_{\alpha \beta}\right)^{2}\right\} \quad \text { on } \quad U_{\alpha} \cap U_{\beta} .
$$

(1) We choose and fix a theta characteristic, or a spin structure for $C$, i.e. a line bundle $K_{C}^{\frac{1}{2}}$ such that $\left(K_{C}^{\frac{1}{2}}\right)^{\otimes 2} \cong K_{C}$.

(2) Let $\left\{\xi_{\alpha \beta}\right\}$ denote the 1-cocycle corresponding to $K_{C}^{\frac{1}{2}}$ with respect to the Möbius coordinate system.

(3) The transition functions for $K_{C}^{\frac{1}{2}}$ are given by

$$
\xi_{\alpha \beta}= \pm\left(c_{\alpha \beta} \cdot z_{\beta}+d_{\alpha \beta}\right) .
$$

The choice of the \pm sign here is exactly an element of $H^{1}(C, \mathbb{Z} / 2 \mathbb{Z})=(\mathbb{Z} / 2 \mathbb{Z})^{2 g}$, which classifies the spin structure of $C$.

Definition 5.1 (Gunning 1967 [23]). A projective coordinate system on $C$ is a coordinate system on which transition function is given by a Möbius transformation. 


$$
C=\bigcup_{\alpha} U_{\alpha}, \quad z_{\alpha} \in U_{\alpha}, \quad z_{\alpha}=\frac{a_{\alpha \beta} \cdot z_{\beta}+b_{\alpha \beta}}{c_{\alpha \beta} \cdot z_{\beta}+d_{\alpha \beta}}, \quad\left[\begin{array}{ll}
a_{\alpha \beta} & b_{\alpha \beta} \\
c_{\alpha \beta} & d_{\alpha \beta}
\end{array}\right] \in S L(2, \mathbb{C}) .
$$

5.2. Hitchin section in rank two. Equipped with the choice of a spin structure for $C$ and the transition functions $\xi_{\alpha \beta}$ for the line bundle $K_{C}^{\frac{1}{2}}$, we define the Hitchin section in rank two.

Recall the Hitchin map for the $S L_{2}(\mathbb{C})$-Higgs bundles sends $\mathcal{M}_{\text {Dol }} \ni(E, \phi) \stackrel{H}{\rightarrow} \operatorname{det}(\phi) \in B$.

Definition 5.2. For each choice of $q \in H^{0}\left(C, K_{C}^{2}\right)=B$, the Hitchin section is the holomorphic Lagrangian inside the Dolbeault moduli space $\mathcal{M}_{\text {Dol }}$, given by

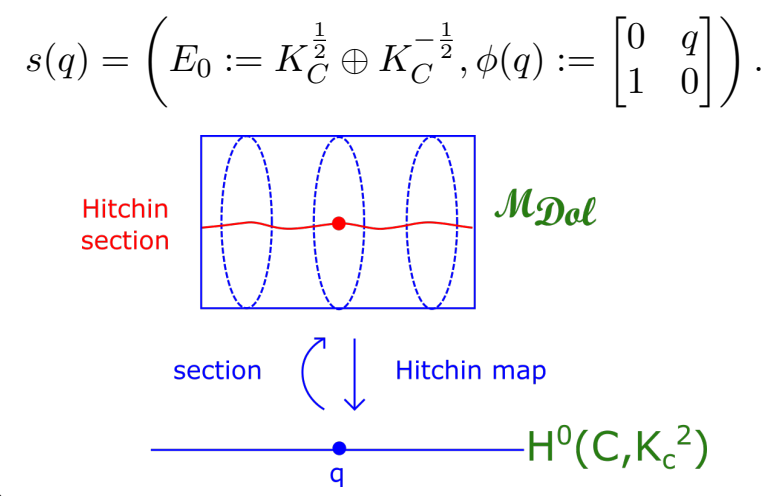

Let $f_{\alpha \beta}=\left(\begin{array}{cc}\xi_{\alpha \beta} & 0 \\ 0 & \xi_{\alpha \beta}^{-1}\end{array}\right)$ be the transition functions of the vector bundle $K_{C}^{\frac{1}{2}} \oplus K_{C}^{-\frac{1}{2}}$. If the quadratic differential $q$ has a local form $\left.q(z)\right|_{U_{\alpha}}=q_{\alpha}\left(z_{\alpha}\right) \cdot d z_{\alpha}^{2}$, then the Higgs field on the Hitchin section $\phi(q)$, which is a matrix valued 1 -form, is given by

$$
\phi_{\alpha}=\left(\begin{array}{cc}
0 & q_{\alpha}\left(z_{\alpha}\right) \cdot d z_{\alpha} \\
d z_{\alpha} & 0
\end{array}\right)
$$

Notice that the Higgs field $\phi(q)$ satisfies the compatibility condition (3.2.3):

$$
\begin{aligned}
f_{\alpha \beta} \cdot \phi_{\beta} \cdot f_{\alpha \beta}^{-1} & =\left(\begin{array}{cc}
\xi_{\alpha \beta} & 0 \\
0 & \xi_{\alpha \beta}^{-1}
\end{array}\right) \cdot\left(\begin{array}{cc}
0 & q_{\beta}\left(z_{\beta}\right) \cdot d z_{\beta} \\
d z_{\beta} & 0
\end{array}\right) \cdot\left(\begin{array}{cc}
\xi_{\alpha \beta}^{-1} & 0 \\
0 & \xi_{\alpha \beta}
\end{array}\right) \\
& =\left(\begin{array}{cc}
0 & \xi_{\alpha \beta}^{2} \cdot q_{\beta}\left(z_{\beta}\right) \cdot d z_{\beta} \\
\xi_{\alpha \beta}^{-2} \cdot d z_{\beta} & 0
\end{array}\right)=\phi_{\alpha} .
\end{aligned}
$$

It follows from noticing $q_{\beta}\left(z_{\beta}\right) d z_{\beta}^{2}=q_{\alpha}\left(z_{\alpha}\right) d z_{\alpha}^{2}$ and $\xi_{\alpha \beta}^{2}=\frac{d z_{\beta}}{d z_{\alpha}}$, concluding that

$$
\xi_{\alpha \beta}^{2} \cdot q_{\beta}\left(z_{\beta}\right) \cdot d z_{\beta}=q_{\beta}\left(z_{\beta}\right) \cdot \frac{d z_{\beta}^{2}}{d z_{\alpha}}=q_{\alpha}\left(z_{\alpha}\right) \cdot d z_{\alpha} .
$$

The stability of the Higgs bundle $s(q)$ with

$$
\phi(q)=\left[\begin{array}{ll}
0 & q \\
1 & 0
\end{array}\right]: K_{C}^{\frac{1}{2}} \oplus K_{C}^{-\frac{1}{2}} \rightarrow K_{C}^{\frac{3}{2}} \oplus K_{C}^{\frac{1}{2}}
$$

is seen as follows. First observe that if $q \neq 0$, then any vector sub-bundle of $E_{0}$, either $0 \oplus K_{C}^{\frac{1}{2}}$ or $K_{C}^{-\frac{1}{2}} \oplus 0$, is not $\phi$ invariant. If $q=0$, then the vector sub-bundle $0 \oplus K_{C}^{-\frac{1}{2}}$ is $\phi$-invariant since it is mapped to zero by $\phi$. However, its slope $1-g$ is negative, since we assume $g \geq 2$. 
Remark 5.3. The Hitchin section (sometimes called the Hitchin component) is a section of the Hitchin fibration 3.2.1 in the sense that it intersects with each fiber of $H$ exactly once. For the case of $S L_{2}(\mathbb{C})$-Higgs bundles (rank 2), it is also a section with respect to the Hitchin map $H$, since $H \circ s=I d_{B}$. However, in general, the Hitchin sections we construct in Section 6 are not the section with respect to the Hitchin map $H$, because $H \circ s \neq I d_{B}$.

For $q \in H^{0}\left(C, K_{C}^{2}\right)$, the differential operator for a Higgs pair on a Hitchin section (5.2) in Theorem 4.1, i.e., the quantum curve $\left.P(x, \hbar \cdot d / d x)\right|_{\hbar=1}=\frac{d^{2}}{d x^{2}}-q(x)$, is not globally defined. This is because unlike the exterior differentiation $d$ which is globally defined, the second order differentiation $d^{2} / d x^{2}$ has no global meaning. However, in the projective coordinate system, the differential equation $\left(\frac{d^{2}}{d x^{2}}-q(x)\right) \psi(x)=0$ makes sense globally on the curve $C$, provided that $\psi$ is a (multi-valued) section of $K_{C}^{-\frac{1}{2}}$. More precisely, with respect to a coordinate change $x=x(u)$, we have

$$
\psi(x(u)) \frac{1}{\sqrt{d x}}=\psi(u) \frac{1}{\sqrt{d u}} \quad \Longleftrightarrow \quad e^{-\frac{1}{2} \log x^{\prime}(u)} \psi(x(u))=\psi(u),
$$

where $x^{\prime}(u)=\frac{d x}{d u}$, and $q(u) d u^{2}=q(x) d x^{2}$. Then

$$
\begin{aligned}
& 0=d u^{2} \cdot e^{\frac{1}{2} \log x^{\prime}(u)}\left[\left(\frac{d}{d u}\right)^{2}-q(u)^{2}\right] \psi(u) \\
& =d u^{2} \cdot e^{\frac{1}{2} \log x^{\prime}(u)}\left[\left(\frac{d}{d u}\right)^{2}-q(u)^{2}\right] e^{-\frac{1}{2} \log x^{\prime}(u)} \psi(x(u)) \\
& =d u^{2} \cdot\left(e^{\frac{1}{2} \log x^{\prime}(u)} \frac{d}{d u} e^{-\frac{1}{2} \log x^{\prime}(u)}\right)^{2} \psi(x(u))-d x^{2} q(x) \psi(x) \\
& =d u^{2} \cdot\left(\frac{d}{d u}-\frac{1}{2} \frac{x^{\prime \prime}}{x^{\prime}}\right)^{2} \psi(x(u))-d x^{2} q(x) \psi(x) \\
& =d u^{2} \cdot\left[\left(\frac{d}{d u}\right)^{2}-\frac{x^{\prime \prime}}{x^{\prime}} \frac{d}{d u}-\frac{1}{2}\left(\left(\frac{x^{\prime \prime}}{x^{\prime}}\right)^{\prime}-\frac{1}{2}\left(\frac{x^{\prime \prime}}{x^{\prime}}\right)^{2}\right)\right] \psi(x(u))-d x^{2} q(x) \psi(x) \\
& =d u^{2} \cdot\left[\left(\psi_{x}(x) x^{\prime}\right)_{u}-\frac{x^{\prime \prime}}{x^{\prime}} \psi_{x}(x) x^{\prime}\right]-d x^{2} q(x) \psi(x) \\
& =\psi_{x x}(x)\left(\frac{d x}{d u}\right)^{2} d u^{2}+d u^{2} \cdot\left(\psi_{x}(x) x^{\prime \prime}-x^{\prime \prime} \psi_{x}(x)\right)-d x^{2} q(x) \psi(x) \\
& =d x^{2} \cdot\left[\left(\frac{d}{d x}\right)^{2}-q(x)\right] \psi(x) .
\end{aligned}
$$

Here, we have used the fact that the Schwarzian derivative

$$
s_{u}(x):=\left(\frac{x^{\prime \prime}(u)}{x^{\prime}(u)}\right)^{\prime}-\frac{1}{2}\left(\frac{x^{\prime \prime}(u)}{x^{\prime}(u)}\right)^{2}
$$

is identically 0 if $x=x(u)=\frac{a_{\alpha \beta} \cdot u+b_{\alpha \beta}}{c_{\alpha \beta} \cdot u+d_{\alpha \beta}},\left[\begin{array}{ll}a_{\alpha \beta} & b_{\alpha \beta} \\ c_{\alpha \beta} & d_{\alpha \beta}\end{array}\right] \in S L(2, \mathbb{C})$. is a Möbius transformation of Definition (5.1). We thus conclude that the quantum curve $\left.P(x, \hbar \cdot d / d x)\right|_{\hbar=1}$ in Theorem 4.1 is globally defined as a twisted $D$-module acting on the sheaf $K_{C}^{-\frac{1}{2}}$. We leave to the interested reader to check the details of this computation. The details of this consideration will be provided in [14, 15]. 
Let us now present an intuitive definition of opers.

Definition 5.4. An oper on an algebraic curve $C$ is a globally defined differential operator of order $r$ acting on $K_{C}^{-\frac{r-1}{2}}$.

Remark 5.5. Importance of Gunning's definition. In a projective coordinate system of $C$, the quantum curve in Theorem $\left.4.1 P(x, \hbar \cdot d / d x)\right|_{\hbar=1}$ is an oper!

5.3. A family of Deligne's $\hbar$-connections. For a Higgs bundle in Definition 5.2, we interpret $P(x, \hbar \cdot d / d x) \psi=0$ as $\nabla^{\hbar}\left[\begin{array}{c}-\hbar \psi^{\prime} \\ \psi\end{array}\right]=0$. Indeed, the quantum curve of the Higgs field

$$
\left(E_{0}=K_{C}^{\frac{1}{2}} \oplus K_{C}^{-\frac{1}{2}}, \phi=\left(\begin{array}{cc}
0 & q(x) \cdot d x \\
d x & 0
\end{array}\right)\right)
$$

is $\left(\hbar^{2} \frac{d^{2}}{d x^{2}}-q(x)\right) \cdot \psi(x, \hbar)=0$. This second order differential equation corresponds to the linear system of ODE, $\left(\hbar \cdot \nabla^{\hbar}\right)\left[\begin{array}{c}-\hbar \cdot \psi \\ \psi\end{array}\right]=0$, where $\nabla^{\hbar}$ is an $\hbar$-deformation family of opers

$$
\nabla^{\hbar}=d+\frac{1}{\hbar} \cdot\left(\begin{array}{cc}
0 & q(x) \cdot d x \\
d x & 0
\end{array}\right) .
$$

Question 5.6. What is the corresponding vector bundle in which this family of connections $\nabla^{\hbar}$ is defined as in Remark 3.4. (3.2.4)?

To answer this question we interpret the complex number $\hbar$ of Theorem 4.1 as an extension class of line bundles $\hbar \in \mathbb{C}=\operatorname{Ext}^{1}\left(K_{C}^{-\frac{1}{2}}, K_{C}^{\frac{1}{2}}\right) \cong H^{1}\left(C, K_{C}\right) \cong H^{0}(C, \mathcal{O})$.

Theorem $5.7([23])$. For every $\hbar \in \mathbb{C}$, there exists a unique extension

$$
0 \rightarrow K_{C}^{\frac{1}{2}} \rightarrow V_{\hbar} \rightarrow K_{C}^{-\frac{1}{2}} \rightarrow 0
$$

such that

(1) the rank 2 vector bundle $V_{\hbar}$ is given by transition functions $\left\{g_{\alpha \beta}^{\hbar}\right\}, g_{\alpha \beta}^{\hbar}:=\left(\begin{array}{cc}\xi_{\alpha \beta} & \hbar \cdot \frac{d \xi_{\alpha \beta}}{d z_{\beta}} \\ 0 & \xi_{\alpha \beta}^{-1}\end{array}\right)$,

(2) $V_{0} \cong K_{C}^{\frac{1}{2}} \oplus K_{C}^{-\frac{1}{2}}$, and

(3) for $\hbar \neq 0$, all the vector bundles $V_{\hbar}$ are isomorphic.

We denote by $V:=\left.V_{\hbar}\right|_{\hbar=1}$ the unique non-trivial extension of $K_{C}^{-\frac{1}{2}}$ by $K_{C}^{\frac{1}{2}}$. We will give a more detail of higher-rank cases in [14, 15].

Proof. We recall that $\xi_{\alpha \beta}$ of 5.1 .2 are transition functions of $K_{C}^{\frac{1}{2}}$.

(1) It is an easy computation to check that $g_{\alpha \beta}^{\hbar}$ are transition functions of $V_{\hbar}$ satisfying the 1-cocycle condition. Denote by $\sigma_{\alpha \beta}=\frac{d \xi_{\alpha \beta}}{d z_{\beta}}$; according to 5.1 .2 , it is a constant. First, we have

$$
g_{\alpha \beta}^{\hbar} \cdot g_{\beta \gamma}^{\hbar}=\left(\begin{array}{cc}
\xi_{\alpha \beta} & \hbar \cdot \sigma_{\alpha \beta} \\
0 & \xi_{\alpha \beta}^{-1}
\end{array}\right) \cdot\left(\begin{array}{cc}
\xi_{\beta \gamma} & \hbar \cdot \sigma_{\beta \gamma} \\
0 & \xi_{\beta \gamma}^{-1}
\end{array}\right)=\left(\begin{array}{cc}
\xi_{\alpha \beta} \cdot \xi_{\beta \gamma} & \hbar \cdot\left(\xi_{\alpha \beta} \sigma_{\beta \gamma}+\xi_{\beta \gamma}^{-1} \sigma_{\alpha \beta}\right) \\
0 & \left(\xi_{\alpha \beta} \cdot \xi_{\beta \gamma}\right)^{-1}
\end{array}\right) .
$$

From the 1-cocycle condition $\xi_{\alpha \beta} \cdot \xi_{\beta \gamma}=\xi_{\alpha \gamma}$ and $\xi_{\alpha \beta}^{2}=\frac{d z_{\beta}}{d z_{\alpha}}$, we claim

$$
\sigma_{\alpha \gamma}=\xi_{\alpha \beta} \sigma_{\beta \gamma}+\xi_{\beta \gamma}^{-1} \sigma_{\alpha \beta} \text {. }
$$


To see this, apply the logarithmic differentiation to $\xi_{\alpha \gamma}=\xi_{\alpha \beta} \cdot \xi_{\beta \gamma}$. We obtain

$$
d z_{\gamma} \cdot \xi_{\alpha \gamma}^{-1} \cdot \frac{d \xi_{\alpha \gamma}}{d z_{\gamma}}=d z_{\beta} \cdot \xi_{\alpha \beta}^{-1} \cdot \frac{d \xi_{\alpha \beta}}{d z_{\beta}}+d z_{\gamma} \cdot \xi_{\beta \gamma}^{-1} \cdot \frac{d \xi_{\beta \gamma}}{d z_{\gamma}} .
$$

Hence

$$
\begin{aligned}
\xi_{\alpha \gamma}^{-1} \cdot \frac{d \xi_{\alpha \gamma}}{d z_{\gamma}} & =\frac{d z_{\beta}}{d z_{\gamma}} \cdot \xi_{\alpha \beta}^{-1} \cdot \sigma_{\alpha \beta}+\xi_{\beta \gamma}^{-1} \cdot \sigma_{\beta \gamma} \\
\sigma_{\alpha \gamma} & =\xi_{\alpha \gamma} \cdot \xi_{\gamma \beta}^{2} \cdot \xi_{\alpha \beta}^{-1} \cdot \sigma_{\alpha \beta}+\xi_{\alpha \gamma} \cdot \xi_{\beta \gamma}^{-1} \cdot \sigma_{\beta \gamma} \\
& =\left(\xi_{\alpha \beta} \cdot \xi_{\beta \gamma}\right) \cdot \xi_{\beta \gamma}^{-2} \cdot \xi_{\alpha \beta}^{-1} \cdot \sigma_{\alpha \beta}+\left(\xi_{\alpha \beta} \cdot \xi_{\beta \gamma}\right) \cdot \xi_{\beta \gamma}^{-1} \cdot \sigma_{\beta \gamma} \\
& =\xi_{\beta \gamma}^{-1} \cdot \sigma_{\alpha \beta}+\xi_{\alpha \beta} \cdot \sigma_{\beta \gamma} .
\end{aligned}
$$

Therefore, $g_{\alpha \beta}^{\hbar} \cdot g_{\beta \gamma}^{\hbar}=g_{\alpha \gamma}^{\hbar}$ in (5.3.3).

(2) Since the matrix $\left.g_{\alpha \beta}^{\hbar}\right|_{\hbar=0}$ is diagonal, the vector bundle it defines splits

$$
V_{0} \cong K_{C}^{\frac{1}{2}} \oplus K_{C}^{-\frac{1}{2}}
$$

(3) For every $\hbar \neq 0$, we show that vector bundle $V_{\hbar}$ is isomorphic to $V$. Indeed, the transition functions $g_{\alpha \beta}^{\hbar}$ and $\left.g_{\alpha \beta}^{\hbar}\right|_{\hbar=1}$ are compatible with the change of trivialization $u_{\alpha}: U_{\alpha} \rightarrow$ $G L_{r}(\mathbb{C})$ (see Section 3.1 )

$$
\begin{aligned}
u_{\alpha} \cdot g_{\alpha \beta}^{\hbar} \cdot u_{\beta}^{-1} & =\left(\begin{array}{cc}
\sqrt{\hbar}^{-1} & 0 \\
0 & \sqrt{\hbar}
\end{array}\right) \cdot\left(\begin{array}{cc}
\xi_{\alpha \beta} & \hbar \cdot \sigma_{\alpha \beta} \\
0 & \xi_{\alpha \beta}^{-1}
\end{array}\right) \cdot\left(\begin{array}{cc}
\sqrt{\hbar} & 0 \\
0 & \sqrt{\hbar}^{-1}
\end{array}\right) \\
& =\left(\begin{array}{cc}
\xi_{\alpha \beta} & \sigma_{\alpha \beta} \\
0 & \xi_{\alpha \beta}^{-1}
\end{array}\right)=\left.g_{\alpha \beta}^{\hbar}\right|_{\hbar=1} .
\end{aligned}
$$

Lemma 5.8. $\nabla^{\hbar}$ in (5.3.1) is a connection on $V_{\hbar}$.

Proof. We first recall equation (3.2.4) for a connection $\nabla^{\hbar}=\left\{d+A_{\alpha}^{\hbar}\right\}$ (5.3.1) on a vector bundle $V_{\hbar}$ given by transition functions $g_{\alpha \beta}^{h}$, where

$$
\begin{aligned}
A_{\alpha}^{\hbar} & =\frac{1}{\hbar}\left(\begin{array}{cc}
0 & q_{\alpha}\left(z_{\alpha}\right) \cdot d z_{\alpha} \\
d z_{\alpha} & 0
\end{array}\right) . \\
A_{\alpha}^{\hbar} & =g_{\alpha \beta}^{\hbar} \cdot A_{\beta}^{\hbar} \cdot\left(g_{\alpha \beta}^{\hbar}\right)^{-1}+g_{\alpha \beta}^{\hbar} \cdot d\left(g_{\alpha \beta}^{\hbar}\right)^{-1} \\
g_{\alpha \beta}^{\hbar} \cdot A_{\beta}^{\hbar} \cdot\left(g_{\alpha \beta}^{\hbar}\right)^{-1} & =\frac{1}{\hbar}\left(\begin{array}{cc}
\xi_{\alpha \beta} & \hbar \cdot \sigma_{\alpha \beta} \\
0 & \xi_{\alpha \beta}^{-1}
\end{array}\right) \cdot\left(\begin{array}{cc}
0 & q_{\beta}\left(z_{\beta}\right) \cdot d z_{\beta} \\
d z_{\beta} & 0
\end{array}\right) \cdot\left(\begin{array}{cc}
\xi_{\alpha \beta}^{-1} & -\hbar \cdot \sigma_{\alpha \beta} \\
0 & \xi_{\alpha \beta}
\end{array}\right) . \\
& =\left(\begin{array}{cc}
\sigma_{\alpha \beta} \cdot \xi_{\alpha \beta}^{-1} & -\hbar \cdot \sigma_{\alpha \beta}^{2}+\frac{1}{\hbar} \xi_{\alpha \beta}^{2} \cdot q_{\beta}\left(z_{\beta}\right) \\
\frac{1}{\hbar} \cdot \xi_{\alpha \beta}^{-2} & -\xi_{\alpha \beta}^{-1} \cdot \sigma_{\alpha \beta}
\end{array}\right) \cdot d z_{\beta} . \\
g_{\alpha \beta}^{\hbar} \cdot d\left(g_{\alpha \beta}^{\hbar}\right)^{-1} & =-\frac{d g_{\alpha \beta}^{\hbar} \cdot\left(g_{\alpha \beta}^{\hbar}\right)^{-1} \cdot d z_{\beta}}{d z_{\beta}} \\
& =-\left(\begin{array}{cc}
\sigma_{\alpha \beta} & \hbar \cdot \frac{d \sigma_{\alpha \beta}}{d z_{\beta}} \\
0 & \frac{d \xi_{\alpha \beta}^{1}}{d z_{\beta}}
\end{array}\right) \cdot\left(\begin{array}{cc}
\xi_{\alpha \beta}^{-1} & -\hbar \cdot \sigma_{\alpha \beta} \\
0 & \xi_{\alpha \beta}
\end{array}\right) \cdot d z_{\beta} \\
& =-\left(\begin{array}{cc}
\sigma_{\alpha \beta} \cdot \xi_{\alpha \beta}^{-1} & -\hbar \cdot \sigma_{\alpha \beta}^{2}+\hbar \cdot \xi_{\alpha \beta} \cdot \frac{d^{2} \xi_{\alpha \beta}}{d z_{\beta}^{2}} \\
0 & -\xi_{\alpha \beta}^{-1} \cdot \sigma_{\alpha \beta}
\end{array}\right) \cdot d z_{\beta} .
\end{aligned}
$$


Relation 5.1 .2 implies $\frac{d^{2} \xi_{\alpha \beta}}{d z_{b}^{2}}=0$. We conclude that

$$
g_{\alpha \beta}^{\hbar} \cdot A_{\beta}^{\hbar} \cdot\left(g_{\alpha \beta}^{\hbar}\right)^{-1}+g_{\alpha \beta}^{\hbar} \cdot d\left(g_{\alpha \beta}^{\hbar}\right)^{-1}=\frac{1}{\hbar} \cdot\left(\begin{array}{cc}
0 & q_{\beta}\left(z_{\beta}\right) \cdot \frac{d z_{\beta}^{2}}{d z_{a}} \\
d z_{\alpha} & 0
\end{array}\right)=\frac{1}{\hbar} \cdot\left(\begin{array}{cc}
0 & q_{\alpha}\left(z_{\alpha}\right) \cdot d z_{a} \\
d z_{\alpha} & 0
\end{array}\right)=A_{\alpha}^{\hbar} .
$$

By fixing a complex structure of the curve $C$ Gunning proved the following isomorphism as affine spaces in [23]

$$
\begin{aligned}
H^{0}\left(C, K_{C}^{2}\right) \cong & \text { moduli space of } S L_{2}(\mathbb{C}) \text {-opers on } C \\
\cong & \text { moduli space of projective coordinate systems on } C \\
& \quad \text { that subordinate the complex structure of } C .
\end{aligned}
$$

Since the space of quadratic differentials $H^{0}\left(C, K_{C}^{2}\right)$ is a vector space, it seems to imply that the holomorphic Lagrangian of opers also inherits an origin, corresponding to $q=0$. Indeed, $\nabla_{\text {unif }}=d+\left(\begin{array}{cc}0 & 0 \\ d x & 0\end{array}\right)$ that we call the uniformizing oper, will play an important role in the next two sections. However, we note that such a choice does not come from algebraic geometry, as we see below.

The computations performed in Definition 5.2 and Lemma 5.8 show that the family $\hbar \cdot \nabla^{\hbar}$, as well as the quantum curve of Theorem 4.1 , is a $\hbar$-connection of Deligne. This is a family of deformations that interpolates a Higgs field $\left.\hbar \cdot \nabla^{\hbar}\right|_{\hbar=0}$ and a genuine connection $\left.\hbar \cdot \nabla^{\hbar}\right|_{\hbar=1}$. We thus conclude that the Dumitrescu-Mulase quantum curve of Theorem 4.1, $\hbar \nabla^{\hbar}$, is an $\hbar$-deformation family of connections constructing a holomorphic passage form a Higgs field on the Hitchin section $\left.\hbar \cdot \nabla^{\hbar}\right|_{\hbar=0}$ to an oper $\left.\hbar \cdot \nabla^{\hbar}\right|_{\hbar=1}$, once we choose a Möbius coordinate system:

$$
\left(K_{C}^{\frac{1}{2}} \oplus K_{C}^{-\frac{1}{2}},\left[\begin{array}{ll}
0 & q \\
1 & 0
\end{array}\right]\right) \stackrel{D M}{\rightarrow}\left(V, d+\left[\begin{array}{cc}
0 & q(x) d x \\
d x & 0
\end{array}\right]\right) .
$$

\section{Hitchin moduli spaces for the Lie group $G=S L_{r}(\mathbb{C})$}

To introduce Gaiotto's correspondence, we need to consider Hitchin moduli spaces for simple complex Lie group $G$. In this paper, we restrict ourselves to the case of $G=S L_{r}(\mathbb{C})$. An $S L_{r}(\mathbb{C})$ Higgs bundle is a pair $(E, \phi)$ consisting of a holomorphic vector bundle $E$ over a smooth projective curve $C$ with a fixed determinant $\operatorname{det}(E)=\wedge^{2} E=\mathcal{O}_{C}$, and a traceless Higgs field $\phi$. We use the same notations in Definition (3.1) in Section 3.1.

- $E, V$ denote holomorphic vector bundles of rank $r$ and degree 0 with trivial determinant.

- $\phi: E \rightarrow E \otimes K_{C}$ is a traceless holomorphic Higgs field.

- $\nabla: V \rightarrow V \otimes K_{C}$ is an irreducible holomorphic connection.

Let $E^{t o p}:=E$ denote the underlying topological structure of the rank $r$ vector bundle $E$, obtained by forgetting its complex structure. Topological complex vector bundles over a compact topological surface are classified by their degrees and ranks, while the complete topological classification of complex vector bundles over a higher dimensional smooth topological manifold is given by their Chern classes. Since $E^{\text {top }}$ has rank $r$ and degree zero, it is topologically isomorphic to the direct sum $\mathcal{O}_{C}^{\oplus r}$ of $r$ copies of the trivial line bundle $\mathcal{O}_{C}$ over $C$.

As mentioned earlier, a classical result of Narasimhan-Seshadri [35] shows that the moduli space $\mathcal{U}_{C}(r, d)$ of stable holomorphic vector bundles of rank $r$ and degree $d$ defined on a smooth projective algebraic curve $C$ is diffeomorphic to the space of projectively flat irreducible unitary connections on $C$. A connection is said to be projectively flat if its curvature takes values in the center of the Lie algebra of the structure group of the vector bundle. For the case of degree 0, there is a one-to-one 
correspondence between stable holomorphic vector bundles and flat irreducible unitary connections. Through the Riemann-Hilbert correspondence, these flat irreducible connections correspond to representations of the fundamental group $\pi_{1}(C)$ into the unitary group modulo conjugation [3, 34]. The equivalence classes of representations form a character variety

$$
\operatorname{Hom}^{\text {irr }}\left(\pi_{1}(C), U_{n}\right) / U_{n} \text {. }
$$

The work of Hitchin [26], Donaldson [8] and Simpson [38] generalizes the above result to the moduli theory of Higgs bundles, holomorphic connections, and complex character varieties. According to this generalization, a stable holomorphic Higgs bundle $(E, \phi)$ of degree 0 corresponds to $(D, \phi, h)$ consisting of the following data that satisfy Hitchin's equations:

- $h$ is a hermitian metric on $E^{\text {top }}$.

- $D$ is a unitary connection on $E^{t o p}$ with respect to the hermitian metric $h$. The connection $D$ decomposes into the holomorphic and antiholomorphic part

$$
D=D^{1,0}+D^{0,1} \text {. }
$$

In terms of a local coordinate $z$ of $C, D$ can locally be given by $D=d+A$ with the exterior differential $d=\partial+\bar{\partial}$, where $\partial=\frac{\partial}{\partial z} \cdot d z$ and $\bar{\partial}=\frac{\partial}{\partial \bar{z}} \cdot d \bar{z}$, and an $r \times r$ skew-hermitian matrix $A$ of 1 -forms on $C$.

- $\phi: E^{t o p} \longrightarrow E^{t o p} \otimes \Omega_{C}^{1}$ is a traceless $r \times r$ matrix of differentiable 1-forms on $C$.

We note that the Cauchy-Riemann part $D^{0,1}$ of the connection $D$ induces a holomorphic structure in $E^{t o p}$, which we denote simply by $E$. The unitarity condition means that the connection $D$ is determined by $D^{0,1}$.

The great discovery of Donaldson [8] is that the stability condition for a Higgs bundle $(E, \phi)$ is the system of non-linear PDEs that Hitchin discovered through the reduction of $4 D$ Yang-Mills self-duality equations. Denote by $F_{D}$ the curvature of the connection $D$,

$$
F_{D}=\frac{1}{2} \cdot[D, D]=\left[D^{1,0}, D^{0,1}\right]
$$

and by $\phi^{\dagger h}$ the adjoint of $\phi$ with respect to the hermitian metric $h$. The following system of non-linear PDEs is known as Hitchin's equations:

$$
\left\{\begin{array}{l}
F_{D}+\left[\phi, \phi^{\dagger h}\right]=0 \\
D^{0,1} \phi=0
\end{array}\right.
$$

For our purpose, it is important that Hitchin's equations 6.0.8 are equivalent to the flatness of the family of connections

$$
D(\zeta):=\frac{1}{\zeta} \cdot \phi+D+\zeta \cdot \phi^{\dagger h}
$$

for all $\zeta \in \mathbb{C}^{*}$. We can see this equivalence as follows. A straightforward calculation shows

$$
\begin{aligned}
{[D(\zeta), D(\zeta)] } & =\frac{1}{\zeta^{2}} \cdot[\phi, \phi]+\zeta^{2} \cdot\left[\phi^{\dagger h}, \phi^{\dagger h}\right]+2 \cdot\left(F_{D}+\left[\phi, \phi^{\dagger h}\right]\right) \\
& +\frac{1}{\zeta} \cdot([\phi, D]+[D, \phi])+\zeta \cdot\left(\left[\phi^{\dagger h}, D\right]+\left[D, \phi^{\dagger h}\right]\right)
\end{aligned}
$$

Clearly (6.0.8) implies the flatness of $D(\zeta)$, because the second equation makes $\phi$ holomorphic with respect to the complex structure of $C$ and the holomorphic structure of $E$. Conversely, from the flatness of $D(\zeta)$, the first equation of (6.0.8) follows from the constant terms with respect to $\zeta$. From the $1 / \zeta^{2}$ terms, we see that $\phi$ contains only $d z$ or $d \bar{z}$ term, and from the $1 / \zeta$ and $\zeta$ terms we see that either $\phi$ or $\phi^{\dagger h}$ is holomorphic. We rename the holomorphic one $\phi$ to obtain (6.0.8). 
We thus have the following correspondences

A stable Higgs bundle $(E, \phi) \longleftrightarrow(D, \phi, h)$ satisfying $(6.0 .8) \longleftrightarrow[D(\zeta), D(\zeta)]=0$ of 6.0 .9$)$.

To deal with three different appearances of complex moduli spaces in the Hitchin theory, we use the terminology gauge theoretical moduli space, denoted by $\mathcal{M}_{\text {Gauge }}$, to describe the differential geometric moduli space of solutions $(D, \phi, h)$ satisfying Hitchin's equations (6.0.8). It is a hyperKähler manifold with $\mathbb{P}^{1}$-worth of complex structures. Customary, we assign the complex structure of $\mathcal{M}_{\mathrm{Dol}}$, the moduli space of stable Higgs bundles, to the origin of $\mathbb{P}^{1}$, and the algebraic structure of $\mathcal{M}_{\mathrm{deR}}$, the moduli space of irreducible holomorphic connections, to $1 \in \mathbb{P}^{1}$. They are both diffeomorphic to $\mathcal{M}_{\text {Gauge }}$.

A particular diffeomorphism, known as the nonabelian Hodge correspondence, between $\mathcal{M}_{D o l}$ and $\mathcal{M}_{\mathrm{deR}}$ is given as follows [8, 26, 38]. Firstly, we assign the flat connection $D(\zeta)$ to $(E, \phi) \in \mathcal{M}_{\text {Dol }}$. Secondly, we define a new holomorphic vector bundle $V=\left(E^{t o p}, D(\zeta=1)^{0,1}\right)$ by using the CauchyRiemann part of the flat connection $D(\zeta)$ at $\zeta=1$. With respect to this complex structure, the $(1,0)$-part of the connection $\nabla:=D(\zeta=1)^{1,0}$ is automatically a holomorphic connection in $V$, since $D(\zeta)$ is flat. Thus we obtain $(V, \nabla) \in \mathcal{M}_{\mathrm{deR}}$.

$$
\mathcal{M}_{\text {Dol }} \ni(E, \phi) \stackrel{N A H}{\longrightarrow}\left(V, \nabla:=D(1)^{1,0}\right) \in \mathcal{M}_{\mathrm{deR}} \cdot
$$

This is a generalization of the classical results of Narasimhan-Seshadri to Higgs bundles. A character variety also comes in to the picture, as the Betti moduli space

$$
\mathcal{M}_{\text {Betti }}:=\operatorname{Hom}^{\text {irr }}\left(\pi_{1}(C), S L_{r}(\mathbb{C})\right) / / S L_{r}(\mathbb{C}) .
$$

The classical unitary group is now replaced by a complex Lie group $G=S L_{r}(\mathbb{C})$. The complex structure of $\mathcal{M}_{\text {Betti }}$ comes from that of the group $S L_{r}(\mathbb{C})$. The Riemann-Hilbert correspondence gives a highly transcendental biholomorphic map between $\mathcal{M}_{\text {deR }}$ and $\mathcal{M}_{\text {Betti }}$. We thus have

$$
\begin{aligned}
\mathcal{M}_{\text {Dol }} & =\text { moduli space of stable holomorphic Higgs bundles }(E, \phi) \text { on } C \text { of rank } r \\
2 & {[\text { Diffeomorphic NAH, Donaldson-Hitchin-Simpson }] } \\
\mathcal{M}_{\text {deR }} & =\text { moduli space of rank r irreducible connections }(V, \nabla) \text { on } C \\
\text { 2\|l } & {[\text { Biholomorphic Riemann-Hilbert }] } \\
\mathcal{M}_{\text {Betti }} & =\operatorname{Hom}^{\text {irr }}\left(\pi_{1}(C), S L_{r}(\mathbb{C})\right) / / S L_{r}(\mathbb{C}) .
\end{aligned}
$$

6.1. Hitchin section for $S L_{r}(\mathbb{C})$-Higgs bundles (principal $s l_{2}(\mathbb{C})$ ). We fix a spin structure $K_{C}^{\frac{1}{2}}$ on $C$ given by transition functions $\left\{\xi_{\alpha \beta}\right\}$. To define a Hitchin section of $G$-Higgs bundles for a simple complex Lie group $G$, we need the notion of Konstant's principal three-dimensional subgroup (TDS) of [29]. For the case of $G=S L_{r}(\mathbb{C})$, it simply comes from the unique $r$-dimensional irreducible representation of $S L_{2}(\mathbb{C})$. The Lie algebra of principal TDS is the linear span $\left\langle X_{+}, X_{-}, H\right\rangle$, where

$$
\begin{aligned}
\text { - } X_{+}: & :=\left[\begin{array}{ccccc}
0 & \sqrt{p_{1}} & 0 & \cdots & 0 \\
0 & 0 & \sqrt{p_{2}} & \cdots & 0 \\
\vdots & \vdots & \vdots & \ddots & \vdots \\
0 & 0 & 0 & \cdots & \sqrt{p_{r-1}} \\
0 & 0 & 0 & \cdots & 0
\end{array}\right], \quad p_{i}:=i(r-i), \\
\text { - } X_{-}:=X_{+}^{t}, &
\end{aligned}
$$




$$
\text { - } H:=\left[X_{+}, X_{-}\right]=\left[\begin{array}{ccccc}
r-1 & 0 & \cdots & 0 & 0 \\
0 & r-3 & \cdots & 0 & 0 \\
\vdots & \vdots & \ddots & \vdots & \vdots \\
0 & 0 & \cdots & -(r-3) & 0 \\
0 & 0 & \cdots & 0 & -(r-1)
\end{array}\right]
$$

Define the split vector bundle $E_{0}:=K_{C}^{\frac{r-1}{2}} \oplus K_{C}^{\frac{r-1}{2}-1} \oplus \ldots \oplus K_{C}^{-\frac{r-1}{2}}$, whose transition function is given by $\left\{\xi_{\alpha \beta}^{H}=\exp \left(H \cdot \log \xi_{\alpha \beta}\right)\right\}$. We note that every $q_{i} \in H^{0}\left(C, K_{C}^{i+1}\right)$ satisfies that $\left.q_{i}\right|_{U_{\alpha}}=\left.q_{i}\right|_{U_{\beta}} \cdot \xi_{\alpha \beta}^{2(i+1)}$.

Now we can generalize Definition 5.2 of Section 5.2 as follows.

Definition 6.1. The Hitchin section is a holomorphic Lagrangian inside $\mathcal{M}_{\text {Dol }}$ consisting of stable Higgs pairs $\left(E_{0}, \phi(q)\right)$ for every $q=\left(q_{1}, \ldots, q_{r-1}\right) \in B=\bigoplus_{i=1}^{r-1} H^{0}\left(C, K_{C}^{i+1}\right)$, where

$$
\phi(q):=X_{-}+\sum_{i=1}^{r-1} q_{i} \cdot X_{+}^{i} .
$$

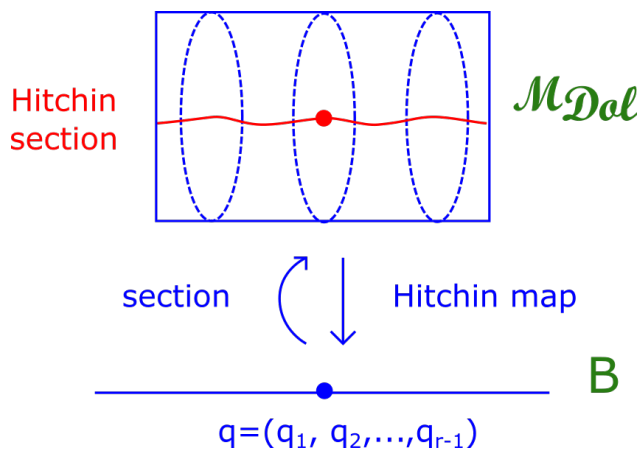

6.2. On a conjecture of Gaiotto. In 2014, Gaiotto [18] conjectured the following.

Conjecture 6.2. Let $\left(E_{0}, \phi\right)$ be a stable Higgs pair on a Hitchin section in $\mathcal{M}_{\mathrm{Dol}}$, and $D(\zeta)$, $\zeta \in \mathbb{C}^{*}$, the corresponding one-parameter family of flat connections. Define a two-parameter family of connections by

$$
D(\zeta, R):=\zeta^{-1} \cdot R \cdot \phi+D+\zeta \cdot R \cdot \phi^{\dagger},
$$

where $R \in \mathbb{R}_{+}$is a positive real number. Then the scaling limit

$$
\lim _{\substack{R \rightarrow 0, \zeta \rightarrow 0 \\ \zeta / R=\hbar}} D(\zeta, R)
$$

exists, and defines an $S L_{r}(\mathbb{C})$-oper for every $\hbar \in \mathbb{C}^{*}$.

The data $(D, \phi, h)$ corresponding to any point $\left(E_{0}, \phi(q)\right)$ on the Hitchin section satisfies Hitchin's equations 6.0.8). Scaling the Higgs field $\phi(q)$ by any real parameter $R \in \mathbb{R}_{+}$does not affect the stability condition. Therefore, the scaled data $(D, R \phi, h)$ corresponding to the pair $\left(E_{0}, R \phi(q)\right) \in$ $\mathcal{M}_{\text {Dol }}$ also satisfies Hitchin's equations. This time, the equation is $R$-twisted:

$$
\left\{\begin{array}{l}
F_{D}+R^{2} \cdot\left[\phi, \phi^{\dagger h}\right]=0 \\
D^{0,1} \phi=0 .
\end{array}\right.
$$

By the same argument as before, the $R$-twisted Hitchin equations are equivalent to the flatness of the two-parameter family of connections $D(\zeta, R)$. 
A surprising fact of this conjecture is that the scaling limit of the differential geometric object $D(\zeta, R)$ is automatically an $\hbar$-family of holomorphic connections defined on an algebraic $\hbar$ deformation family of filtered vector bungles, generalizing the extension

$$
0 \rightarrow K_{C}^{\frac{1}{2}} \rightarrow V_{\hbar} \rightarrow K_{C}^{-\frac{1}{2}} \rightarrow 0 .
$$

Naïvely, it looks that the scaling limit of (6.2.1) is simply $D+\frac{1}{\hbar} \phi$. There is a problem here, because the hermitian metric $h$ that solves $(6.2 .2)$ explodes as $R \rightarrow 0$. Since the $h$-unitary connection $D$ also depends on $h$, the limit of $D$ does not make sense as $R$ tends to 0 .

Theorem 6.3 (Dumitrescu, Fredrickson, Kydonakis, Mazzeo, Mulase, Neitzke, [9]). Conjecture 6.2 holds for an arbitrary simple and simply connected complex Lie group $G$.

6.3. Sketch of the proof in rank two. We present here the main steps to prove Theorem 6.3 for the case of $G=S L_{2}(\mathbb{C})$. We use the basis

$$
\left\langle X_{+}=\left[\begin{array}{ll}
0 & 1 \\
0 & 0
\end{array}\right], X_{-}=\left[\begin{array}{ll}
0 & 0 \\
1 & 0
\end{array}\right], H=\left[\begin{array}{cc}
1 & 0 \\
0 & -1
\end{array}\right]\right\rangle
$$

for $s l_{2}(\mathbb{C})$ to simplify our calculations. Their commutation relations are

$$
\left[X_{+}, X_{-}\right]=H, \quad\left[H, X_{ \pm}\right]= \pm 2 X_{ \pm} .
$$

Step 1. We first notice that a hermitian metric on the canonical bundle $K_{C}$ naturally comes from a hermitian metric on the curve $C$ itself. Since we start from a Higgs pair on a Hitchin section with vector bundle $E_{0}=K_{C}^{\frac{1}{2}} \oplus K_{C}^{-\frac{1}{2}}$, a fiber metric is determined by a hermitian metric on $C$. Recall that

$$
\text { A Higgs bundle } \left.\left(E_{0}, \phi\right) \text { on the Hitchin section } \longrightarrow(D, \phi, h) \text { satisfying } 6.0 .8\right) \text {. }
$$

A choice of a hermitian metric on $C$ determines the fiber metric $h$, and hence the unitary connection $D$. Thus we wish to see how it translates into Hitchin's equations.

Step 2. We start from a complex structure on $C$ with a holomorphic local parameter $z$, together with a hermitian metric

$$
g=\lambda^{2} \cdot d z \cdot d \bar{z}
$$

on $C$, where $\lambda$ is a positive real function depending on $R$. The hermitian metric on $C$ is the same as the fiber metric of the tangent bundle of $C$, which is $K_{C}^{-1}$. Hence $\lambda$ naturally gives a fiber metric of $K_{C}^{-\frac{1}{2}}$. Therefore, the hermitian metric on the split vector bundle $E_{0}=K_{C}^{\frac{1}{2}} \oplus K_{C}^{-\frac{1}{2}}$ is given by the matrix $h=\left[\begin{array}{cc}\lambda^{-1} & 0 \\ 0 & \lambda .\end{array}\right]$. Then $D$ becomes the Chern connection $D=D^{1,0}+D^{0,1}$, where $D^{0,1}=\bar{\partial}$ and $D^{1,0}=\partial+h^{-1} \cdot \partial h$. In terms of $\lambda$, we have

$$
D=d+h^{-1} \cdot \partial h=d-\partial \log \lambda \cdot\left[\begin{array}{cc}
1 & 0 \\
0 & -1
\end{array}\right]=d-\partial \log \lambda \cdot H .
$$

Step 3. Let us introduce a Higgs field

$$
\phi=\phi(q)=\left[\begin{array}{ll}
0 & q \\
1 & 0
\end{array}\right] \cdot d z=\left(X_{-}+q \cdot X_{+}\right) \cdot d z
$$

so that $\left(E_{0}, \phi(q)\right)$ is on the Hitchin section, where $q \in H^{0}\left(C, K_{C}^{2}\right)$. The hermitian conjugate of the Higgs field is calculated by

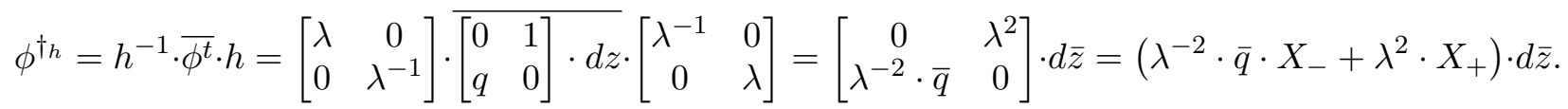


Step 4. Since we have identified all the ingredients, we can now write the two-parameter family of connections in this local coordinate as

$$
\begin{aligned}
D(\zeta, R) & =\frac{R}{\zeta} \cdot\left[\begin{array}{ll}
0 & q \\
1 & 0
\end{array}\right] \cdot d z+d-\partial \log \lambda \cdot\left[\begin{array}{cc}
1 & 0 \\
0 & -1
\end{array}\right] \cdot d z+R \cdot \zeta \cdot\left[\begin{array}{cc}
0 & \lambda^{2} \\
\bar{q} \cdot \lambda^{-2} & 0
\end{array}\right] \cdot d \bar{z} \\
& =d+\frac{R}{\zeta} \cdot d z \cdot\left(X_{-}+q \cdot X_{+}\right)-\partial \log \lambda \cdot d z \cdot H+R \cdot \zeta \cdot d \bar{z} \cdot\left(\bar{q} \cdot \lambda^{-2} \cdot X_{-}+\lambda^{2} \cdot X_{+}\right) .
\end{aligned}
$$

Step 5. A simple calculation shows that the coefficient of $H$ in the flatness condition

$$
[D(\zeta, R), D(\zeta, R)]=0
$$

of $D(\zeta, R)$ yields

$$
\begin{aligned}
0 & =[d,-\partial \log \lambda \cdot d z \cdot H]+R^{2} \cdot d z \wedge d \bar{z} \cdot\left(\lambda^{2} \cdot\left[X_{-}, X_{+}\right]+q \cdot \bar{q} \cdot \lambda^{-2} \cdot\left[X_{+}, X_{-}\right]\right) \\
& =-d \partial \log \lambda \cdot d z \cdot H+R^{2} \cdot d z \wedge d \bar{z} \cdot\left(-\lambda^{2} \cdot H+q \cdot \bar{q} \cdot \lambda^{-2} \cdot H\right) \\
& =\left(\bar{\partial} \partial \log \lambda+R^{2} \cdot\left(\lambda^{-2} \cdot q \cdot \bar{q}-\lambda^{2}\right)\right) \cdot d z \wedge d \bar{z}
\end{aligned}
$$

Therefore, we obtain

$$
\bar{\partial} \partial \log \lambda+R^{2} \cdot\left(\lambda^{-2} \cdot q \cdot \bar{q}-\lambda^{2}\right)=0 .
$$

We thus conclude that the flatness condition for the two-parameter family of connections $D(\zeta, R)$ gives the harmonicity condition 6.3.1 for the hermitian metric $\lambda$.

Step 6. For $q=0$, i.e., $\phi=X_{-}$, the harmonicity equation 6.3.1 becomes

$$
\bar{\partial} \partial \log \lambda-R^{2} \cdot \lambda^{2}=0
$$

which can be solved explicitly. We obtain

$$
\lambda_{0}=\frac{1}{R} \cdot \frac{i}{z-\bar{z}}
$$

Let us denote by

$$
\lambda_{\natural}=\frac{i}{z-\bar{z}}=\frac{1}{2 \cdot y},
$$

where $z=x+i y$. The corresponding hermitian metric is then

$$
g_{\natural}=\frac{d z \cdot d \bar{z}}{4 \cdot y^{2}},
$$

whose Gaussian curvature is

$$
K:=-\frac{4}{\lambda_{\natural}^{2}} \cdot \partial \bar{\partial} \log \lambda_{\natural}=-4 .
$$

Indeed, $g_{\natural}$ is the globally defined constant curvature metric on the upper half plane $\mathbb{H}$, which is invariant under the action of $P S L_{2}(\mathbb{R})=\operatorname{Aut}(\mathbb{H})$. Since we are dealing with a Riemann surface $C$ of genus $g \geq 2$, its universal covering is $\mathbb{H}$, and we have a non-canonical isomorphism $C \cong \mathbb{H} / \pi_{1}(C)$, where $\pi_{1}(C)$ acts on $\mathbb{H}$ through a representation $\rho: \pi_{1}(C) \longrightarrow S L_{2}(\mathbb{R})$. By inducing a metric by the push-forward of the covering map $\mathbb{H} \longrightarrow C$, we conclude that the harmonicity equation (6.3.2) can be solved globally on $C$ with the hyperbolic metric on $C$ of constant curvature $-4 R^{2}$.

Since $\frac{\zeta}{R}=\hbar$, we obtain

$$
\begin{aligned}
D(\zeta, R) & =d+\frac{1}{\hbar} \cdot\left[\begin{array}{ll}
0 & 0 \\
1 & 0
\end{array}\right] \cdot d z-\partial \log \lambda_{\natural} \cdot\left[\begin{array}{cc}
1 & 0 \\
0 & -1
\end{array}\right] d z+\hbar \cdot\left[\begin{array}{cc}
0 & \lambda_{\natural}^{2} \\
0 & 0
\end{array}\right] d \bar{z} \\
& =d+\frac{1}{\hbar} \cdot d z \cdot X_{1}-\partial \log \lambda_{\natural} \cdot d z \cdot H+\hbar \cdot \lambda_{\natural}^{2} \cdot d \bar{z} \cdot X_{+},
\end{aligned}
$$


which does not depend on $R$.

Step 7. The case when $q \neq 0$ in general. We remark that any hermitian metric compatible with the complex structure of $C$ is conformal to the constant curvature metric $g_{\natural}$. Therefore, we can write

$$
\lambda=\lambda_{0} \cdot e^{f(R)}=\frac{\lambda_{\natural}}{R} \cdot e^{f(R)}
$$

with a conformal factor $e^{f(R)}$ depending on a real valued function $f(R)$ on $C$. We plug this expression into (6.3.1) and apply the implicit function theorem to yield that $f$ is real analytic, and more significantly, that $f(R)=f_{4} \cdot R^{4}+$ higher order terms. This implies

$$
\lambda^{-2}=\frac{R^{2}}{\lambda_{\natural}^{2}} \cdot e^{-2 f(R)}=\frac{R^{2}}{\lambda_{\natural}^{2}}+\mathcal{O}\left(R^{6}\right), \quad \lambda=\frac{\lambda_{\natural}}{R}+\mathcal{O}\left(R^{3}\right), \quad \partial \log \lambda=\partial \log \lambda_{\natural}+\mathcal{O}\left(R^{4}\right) .
$$

Therefore, we obtain the scaling limit as

$$
\begin{aligned}
D(\zeta, R) & =d+\frac{1}{\hbar} \cdot\left[\begin{array}{ll}
0 & q \\
1 & 0
\end{array}\right] \cdot d z-\partial \log \lambda \cdot\left[\begin{array}{cc}
1 & 0 \\
0 & -1
\end{array}\right] \cdot d z+R^{2} \cdot \hbar\left[\begin{array}{cc}
0 & \lambda^{2} \\
\bar{q} \cdot \lambda^{-2} & 0
\end{array}\right] \cdot d \bar{z} \\
& =d+\frac{1}{\hbar} \cdot\left[\begin{array}{ll}
0 & q \\
1 & 0
\end{array}\right] \cdot d z-\partial \log \lambda_{\natural} \cdot\left[\begin{array}{cc}
1 & 0 \\
0 & -1
\end{array}\right] \cdot d z+\mathcal{O}\left(R^{4}\right) \cdot\left[\begin{array}{cc}
1 & 0 \\
0 & -1
\end{array}\right] \cdot d z \\
& +\hbar \cdot\left[\begin{array}{cc}
0 & \lambda_{\natural}^{2}+\mathcal{O}\left(R^{4}\right) \\
\bar{q} \cdot \frac{R^{4}}{\lambda_{\natural}^{2}}+\mathcal{O}\left(R^{8}\right) & 0
\end{array}\right] \cdot d \bar{z} \\
& \frac{R \rightarrow 0, \zeta \rightarrow 0}{\zeta / R=\hbar} d+\frac{1}{\hbar} \cdot\left[\begin{array}{cc}
0 & q \\
1 & 0
\end{array}\right] \cdot d z-\partial \log \lambda_{\natural} \cdot\left[\begin{array}{cc}
1 & 0 \\
0 & -1
\end{array}\right] \cdot d z+\hbar \cdot\left[\begin{array}{cc}
0 & \lambda_{\natural}^{2} \\
0 & 0
\end{array}\right] \cdot d \bar{z} \\
& =d+\frac{1}{\hbar} \cdot d z \cdot\left(X_{-}+q \cdot X_{+}\right)-\partial \log \lambda_{\natural} \cdot d z \cdot H+\hbar \cdot \lambda_{\natural}^{2} \cdot d \bar{z} \cdot X_{+} \cdot
\end{aligned}
$$

We can see that the only dependence on the quadratic differential $q$ is in the form of $\phi(q)$.

\section{The limit oper of Gaiotto's correspondence and the quantum Curve}

In this section we will prove that the scaling $\operatorname{limit}_{R, \zeta \rightarrow 0, \frac{\zeta}{R}=\hbar} D(\zeta, R)$ is an oper.

A surprising fact is that the limit oper of Theorem 6.3 [9] is gauge equivalent to $\nabla^{\hbar}=d+\frac{1}{\hbar} \cdot \phi$ in the Möbius coordinate system obtained by the uniformization of the base curve $C$. In other words, the limit oper of Gaiotto correspondence in rank two is the quantum curve of Theorem 4.1 ([10]). We use Theorem 5.7 and Lemma 5.8 of Section 5.3 to imply that the scaling limit of Gaiotto's correspondence is actually an oper. For the sake of completeness, we include detailed computations here.

Proposition 7.1 (Gauge transform of the scaling Limit). The limit expression of (6.3.7),

$$
D(\hbar):=d+\frac{1}{\hbar} \cdot d z \cdot\left(X_{-}+q \cdot X_{+}\right)-\partial \log \lambda_{\natural} \cdot d z \cdot H+\hbar \cdot \lambda_{\natural}^{2} \cdot d \bar{z} \cdot X_{+},
$$

is gauge equivalent to an oper

$$
\nabla^{\hbar}:=d+\frac{1}{\hbar} \cdot\left[\begin{array}{ll}
0 & q \\
1 & 0
\end{array}\right] \cdot d z=d+\frac{1}{\hbar} \cdot \phi(q)
$$

Here, $\lambda_{\natural}=\frac{i}{z-\bar{z}}$ is the local expression of the hermitian metric of constant curvature -4 on $C$. If we introduce a Möbius coordinate system on $C$ induced by the uniformization covering $\mathbb{H} \longrightarrow C$, then (7.0.8) determines a globally defined oper on $C$. 
Proof. The claim is that $\nabla^{\hbar}=g \cdot D(\hbar) \cdot g^{-1}$ with the gauge transformation

$$
g=\left[\begin{array}{cc}
1 & \hbar \cdot \partial \log \lambda_{\natural} \\
0 & 1
\end{array}\right]=e^{\hbar \cdot \partial \log \lambda_{\natural} \cdot X_{+}} .
$$

For brevity, let us denote $a=\partial \log \lambda_{\natural}$ and $b=\lambda_{\natural}^{2}$. Then

$$
D(\hbar)=d+\frac{1}{\hbar} \cdot d z \cdot\left(X_{-}+q \cdot X_{+}\right)-a \cdot d z \cdot H+\hbar \cdot b \cdot d \bar{z} \cdot X_{+}, \quad g=e^{\hbar \cdot a \cdot X_{+}} .
$$

Recall that for any elements $A$ and $B$ of a Lie algebra and a central parameter $\epsilon$, we have the adjoint formula

$$
e^{\epsilon A} \cdot B \cdot e^{-\epsilon A}=\sum_{n=0}^{\infty} \frac{1}{n !} \epsilon^{n} \cdot \operatorname{ad}_{A}^{n}(B),
$$

where

$$
\operatorname{ad}_{A}^{n}(B):=\overbrace{[A,[A,[\cdots,[A}^{n}, B] \cdots]]] .
$$

From the commutation relations of the basis for $s l_{2}(\mathbb{C})$, we see that

$$
g \cdot X_{-} \cdot g^{-1}=X_{-}+\hbar \cdot a \cdot\left[X_{+}, X_{-}\right]+\frac{1}{2}(\hbar \cdot a)^{2} \cdot\left[X_{+},\left[X_{+}, X_{-}\right]\right]=X_{-}+\hbar \cdot a \cdot H-(\hbar \cdot a)^{2} \cdot X_{+} \cdot
$$

Therefore,

$$
g \cdot \frac{1}{\hbar} \cdot d z \cdot\left(X_{-}+q \cdot X_{+}\right) \cdot g^{-1}=\left(\frac{1}{\hbar} \cdot\left(X_{-}+q \cdot X_{+}\right)+a \cdot H-\hbar \cdot a^{2} \cdot X_{+}\right) \cdot d z .
$$

Similarly,

$$
g \cdot H \cdot g^{-1}=H+\hbar \cdot a \cdot\left[X_{+}, H\right]=H-2 \hbar \cdot a \cdot X_{+},
$$

which yields

$$
-g \cdot a \cdot d z \cdot H \cdot g^{-1}=-a \cdot d z \cdot H+2 \hbar \cdot a^{2} \cdot d z \cdot X_{+} .
$$

It is obvious that

$$
g \cdot \hbar \cdot b \cdot d \bar{z} \cdot X_{+} \cdot g^{-1}=\hbar \cdot b \cdot d \bar{z} \cdot X_{+}
$$

Finally, we calculate

$$
\begin{aligned}
g \cdot d \cdot g^{-1} & =d-\hbar \cdot d a \cdot X_{+} \\
& =d-\hbar \cdot(\partial a \cdot d z+\bar{\partial} \cdot a \cdot d \bar{z}) \cdot X_{+} \\
& =d-\hbar \cdot d z \cdot \partial^{2} \log \lambda_{\natural} \cdot X_{+}-\hbar \cdot d \bar{z} \cdot \bar{\partial} \partial \log \lambda_{\natural} \cdot X_{+} \\
& =d+\hbar \cdot d z \cdot \frac{\left(\partial \lambda_{\natural}\right)^{2}-\lambda_{\natural} \cdot \partial^{2} \lambda_{\natural}}{\lambda_{\natural}^{2}} \cdot X_{+}-\hbar \cdot d \bar{z} \cdot \lambda_{\natural}^{2} \cdot X_{+},
\end{aligned}
$$

where we used the constant curvature property 6 6.3.5 in the last step.

Adding all together, we obtain the gauge transformation formula

$$
\begin{aligned}
g \cdot D(\hbar) \cdot g^{-1}=d+ & \left(\frac{1}{\hbar} \cdot\left(X_{-}+q \cdot X_{+}\right)+\partial \log \lambda_{\natural} \cdot H-\hbar \cdot\left(\partial \log \lambda_{\natural}\right)^{2} \cdot X_{+}\right) \cdot d z+ \\
& +\hbar \cdot d z \cdot \frac{\left(\partial \lambda_{\natural}\right)^{2}-\lambda_{\natural} \cdot \partial^{2} \lambda_{\natural}}{\lambda_{\natural}^{2}} \cdot X_{+}+\left(\hbar \cdot b \cdot d \bar{z}-\hbar \cdot d \bar{z} \cdot \lambda_{\natural}^{2}\right) \cdot X_{+}- \\
& \quad-\partial \log \lambda_{\natural} \cdot d z \cdot H+2 \hbar \cdot\left(\partial \log \lambda_{\natural}\right)^{2} \cdot d z \cdot X_{+} \\
=d & +\frac{1}{\hbar} \cdot\left(X_{-}+q \cdot X_{+}\right) \cdot d z+\hbar \cdot d z \cdot \frac{2 \cdot\left(\partial \lambda_{\natural}\right)^{2}-\lambda_{\natural} \cdot \partial^{2} \lambda_{\natural}}{\lambda_{\natural}^{2}} \cdot X_{+}
\end{aligned}
$$




$$
=d+\frac{1}{\hbar} \cdot \phi(q)
$$

Here, we use the fact that $2 \cdot\left(\partial \lambda_{\natural}\right)^{2}-\lambda_{\natural} \cdot \partial^{2} \lambda_{\natural}=0$, which follows from the expression 6.3.4).

The computation we have shown above is only for $S L_{2}(\mathbb{C})$. Yet it is valid for proving vastly general Theorem 6.3. The key idea is to use Kostant's principal TDS [29], replacing the basis $\left\langle H, X_{ \pm}\right\rangle$by the one for TDS. Then almost exactly the same formulas hold for the general situation. Here, we only indicate the oper we obtain through Gaiotto's scaling limit for the case of $S L_{r}(\mathbb{C})$ Higgs bundles. We will give a geometric definition of oper that generalizes Gunning's Definition encaptured by Theorem 5.7 from rank two to arbitrary rank $r$.

Definition 7.2 (Beilinson-Drinfeld, 1993). Let $V$ be a holomorphic vector bundle of rank $r$ and degree 0 . An $S L_{r}(\mathbb{C})$-oper is a pair $(V, \nabla) \in \mathcal{M}_{\text {deR }}$ satisfying the following conditions:

(1) There is a global filtration $0=F_{r} \hookrightarrow F_{r-1} \hookrightarrow \ldots \hookrightarrow F_{0}=V$ in $V$.

(2) Griffiths transversality. The connection $\nabla$ induces a map $\left.\nabla\right|_{F_{i+1}}: F_{i+1} \rightarrow F_{i} \otimes K_{C}$ for every $i=0, \ldots, r-1$.

(3) $\left.\nabla\right|_{F_{i+1}}$ induces an $\mathcal{O}_{C}$-linear isomorphism $F_{i+1} / F_{i+2} \cong F_{i} / F_{i+1} \otimes K_{C}$ for every $i$.

Let $\left(E_{0}, \phi(q)\right)$ be a point on the Hitchin section of Section 6.1. Then Gaiotto's scaling limit produces an $\hbar$-family of opers $\left(V_{\hbar}, \nabla^{\hbar}\right)$ defined as follows. First, we choose once and for all the Möbius coordinate system associated with the uniformization mentioned above.

- $V_{\hbar}$ is given by the transition function $\left\{f_{\alpha \beta}^{\hbar}\right\}$, where

$$
f_{\alpha \beta}^{\hbar}=\exp \left(H \cdot \log \xi_{\alpha \beta}\right) \cdot \exp \left(\hbar \cdot \frac{d \log \xi_{\alpha \beta}}{d z_{\beta}} \cdot X_{+}\right) .
$$

- The connection is defined by

$$
\nabla^{\hbar}:=d+\frac{1}{\hbar} \cdot \phi(q)
$$

Note that this definition is globally valid with respect to a Möbius coordinate system.

- $V_{0}=K_{C}^{\frac{r-1}{2}} \oplus \ldots \oplus K_{C}^{-\frac{r-1}{2}}=E_{0}$, since $f_{\alpha \beta}^{\hbar=0}=\exp \left(H \cdot \log \xi_{\alpha \beta}\right)=\xi_{\alpha \beta}^{H}$.

- There is a unique filtration in the vector bundle $V_{\hbar}$ with $F_{r-1}=K_{C}^{\frac{r-1}{2}}$ that satisfies the conditions of Definition 7.2.

- The vector bundles $V_{\hbar}$ are isomorphic for all $\hbar \neq 0$.

We refer to [14, 15] for more detail.

\section{Conclusion}

We emphasize again that the nonabelian Hodge correspondence is a diffeomorphism between $\mathcal{M}_{\text {Dol }}$ and $\mathcal{M}_{\mathrm{deR}}$. $([8,26,38)$. Conjecture 6.2 of Gaiotto realizes a holomorphic point by point correspondence between two holomorphic Lagrangians, the Hitchin section in $\mathcal{M}_{\text {Dol }}$ and the moduli space of opers in $\mathcal{M}_{\mathrm{deR}}$. Since the quantum curve should depend holomorphically on the spectral curve, we consider the Gaiotto correspondence as the desired construction of quantum curves.

- Donaldson, Hitchin, Simpson Nonabelian-Hodge correspondence

$$
\mathcal{M}_{\text {Dol }} \stackrel{\text { diffeomorphism }}{\longrightarrow} \mathcal{M}_{\text {deR }}
$$


- Gaiotto's conjecture gives a holomorphic correspondence between Lagrangians

$$
\text { Hitchin Section } \stackrel{\text { holomorphic }}{\longrightarrow} \text { moduli space of opers }
$$

Quantization is never unique. Yet the Catalan example we presented earlier clearly shows why we are interested in the unique process of quantization. A quantum curve quantizes the B-model geometry, which provides a generating function of genus $g$ A-models for all $g$. Thus we wish a unique quantization result.

Starting from a Hitchin spectral curve, we identify the Higgs bundle on the Hitchin section. This is unique, once the spin structure of the curve $C$ is chosen. Then the correspondence given by Gaiotto's scaling limit constructs, again, a unique oper in the moduli space of holomorphic connections on $C$. Thus the process from the spectral curve to the quantum curve (oper) is unique, and depends holomorphically on the moduli of spectral curves, when the complex structure of $C$ is fixed.

We present below a local picture of the two Lagrangians inside the Dolbeault moduli space together with their images under the nonabelian-Hodge and Gaiotto's correspondences. In the figure, $V_{1}=\left.V_{\hbar}\right|_{\hbar=1}$. The picture does not show the global relations between various Lagrangians in the moduli spaces. For example, the $S L_{r}(\mathbb{R})$-Hitchin component in $\mathcal{M}_{\mathrm{deR}}$ and the oper moduli space intersects infinitely many times. Only locally they intersect at a point, here at $\left(V_{1}, d+X_{-}\right)$.

Gaiotto Correspondence $=$ Canonical Biholomorphic Map

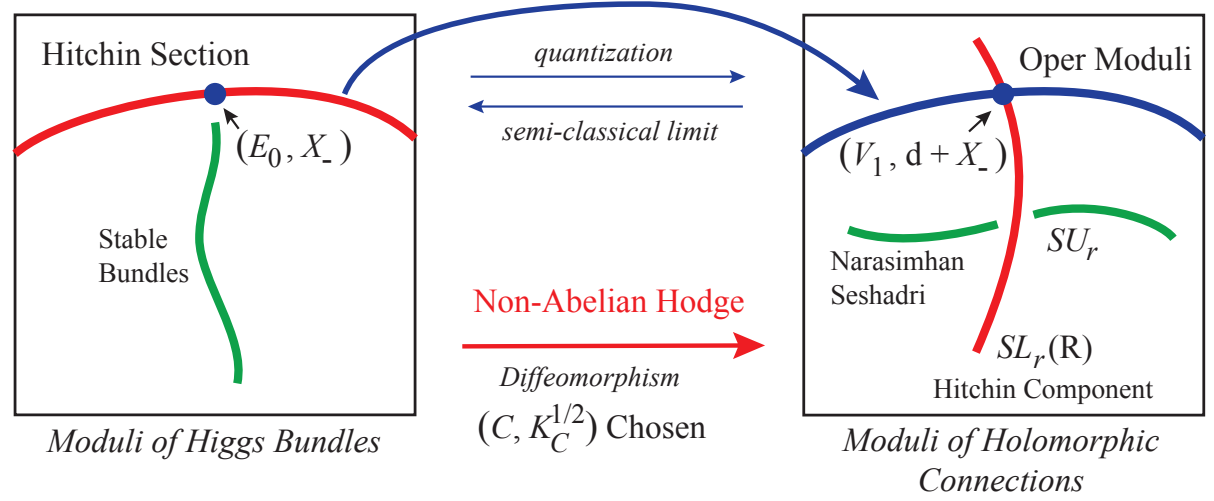




\section{REFERENCES}

[1] M. Aganagic, R. Dijkgraaf, A. Klemm, M. Mariño, and C. Vafa, Topological Strings and Integrable Hierarchies,[arXiv:hep-th/0312085], Commun. Math. Phys. 261, 451-516 (2006).

[2] M.F. Atiyah, Vector bundles over an elliptic curve, Proc. London Math. Soc. (3), 7:414452, 1957.

[3] M.F. Atiyah and R. Bott, The Yang-Mills equations over Riemann surfaces, Phil. Trans. Royal Soc. London A 308, 523-615 (1982).

[4] A. Beilinson and V. Drinfeld, Opers, arXiv:math/0501398v1 [math.AG] (2005).

[5] R. Dijkgraaf, L. Hollands, and P. Sułkowski, Quantum curves and D-modules, Journal of High Energy Physics 0810.4157, 1-58 (2009).

[6] R. Dijkgraaf, L. Hollands P. Sułkowski, and C. Vafa, Supersymmetric gauge theories, intersecting branes and free Fermions, Journal of High Energy Physics 0802.106, (2008).

[7] R. Dijkgraaf, E. Verlinde, and H. Verlinde, Loop equations and Virasoro constraints in non-perturbative twodimensional quantum gravity, Nucl. Phys. B348, 435-456 (1991).

[8] S.K. Donaldson, A new proof of a theorem of Narasimhan and Seshadri, Journal of Differential Geometry, 18 (2): 269277, (1983).

[9] O. Dumitrescu L. Fredrickson, G. Kydonakis, R. Mazzeo, M. Mulase and A. Neitzke, Opers versus nonabelian Hodge http://arxiv.org/pdf/1607.02172v1.pdf, under review.

[10] O. Dumitrescu and M. Mulase, Quantum curves for Hitchin fibrations and the Eynard-Orantin theory, Lett. Math. Phys. 104, 635-671 (2014).

[11] O. Dumitrescu and M. Mulase, Quantization of spectral curves for meromorphic Higgs bundles through topological recursion, http://arxiv.org/pdf/1411.1023v1.pdf, under review.

[12] O. Dumitrescu and M. Mulase, Edge-contraction on dual ribbon graphs, 2D TQFT, and the mirror of orbifold Hurwitz numbers, http://arxiv.org/pdf/1508.05922v1.pdf, under review in Journal of Algebra.

[13] O. Dumitrescu and M. Mulase, Lectures on the topological recursion for Hitchin spectral curves and quantization, http://arxiv.org/pdf/1509.09007v1.pdf to appear in Lecture Notes Series, Institute for Mathematical Sciences, National University of Singapore.

[14] O. Dumitrescu and M. Mulase, Lectures on topological quantum field theory and character varieties, Lecture Notes.

[15] O. Dumitrescu and M. Mulase, Weyl quantization of Hitchin spectral curves and opers, "Proceedings of the 2016 AMS von Neumann Symposium," Proceedings of Symposia in Pure Mathematics, American Mathematical Society.

[16] O. Dumitrescu, M. Mulase, B. Safnuk, A. Sorkin The Spectral Curve of the Eynard-Orantin Recursion via the Laplace Transform in Algebraic and Geometric Aspects of Integrable Systems and Random Matrices, Dzhamay, Maruno and Pierce, Eds. Contemporary Mathematics 593, 263-315 (2013)

[17] B. Eynard and N. Orantin, Invariants of algebraic curves and topological expansion, Communications in Number Theory and Physics 1, 347-452 (2007).

[18] D. Gaiotto, Opers and TBA, arXiv:1403.6137 [hep-th], (2014).

[19] D. Gaiotto, G.W. Moore, and A. Neitzke,Wall-crossing, Hitchin systems, and the WKB approximation, arXiv:0907.3987 [hep-th] (2009).

[20] A. Grothendieck, Sur la classification des fibres holomorphes sur la sphère de Riemann, Am. J. Math., 79:121138, 1957

[21] A. Grothendieck, Esquisse d'un programme, (1984).

[22] S. Gukov and P. Sułkowski, A-polynomial, B-model, and quantization, arXiv:1108.0002v1 [hep-th] (2011).

[23] R.C. Gunning, Special coordinate coverings of Riemann surfaces, Math. Annalen 170, 67-86 (1967).

[24] J. L. Harer, The cohomology of the moduli space of curves, in Theory of Moduli, Montecatini Terme, 1985 (Edoardo Sernesi, ed.), Springer-Verlag, 1988, pp. 138-221.

[25] J. Harer and D. Zagier, The Euler characteristic of the moduli space of curves, Inventiones Mathematicae 85, 457-485 (1986).

[26] N.J. Hitchin, The self-duality equations on a Riemann surface, Proc. London Math. Soc. (Ser. 3) 55, 59-126 (1987).

[27] M. Kontsevich, Intersection theory on the moduli space of curves and the matrix Airy function, Communications in Mathematical Physics 147, 1-23 (1992).

[28] M. Kontsevich and Y. Manin, Gromov-Witten classes, quantum cohomology, and enumerative geometry, Communications in Mathematical Physics 164, 525-562 (1994).

[29] B. Kostant, The principal three-dimensional subgroup and the Betti numbers of a complex simple Lie group, Amer. J. Math., 81, 973-1032 (1959). 
[30] M. Mulase and M. Penkava, Ribbon graphs, quadratic differentials on Riemann surfaces, and algebraic curves defined over $\overline{\mathbb{Q}}$, The Asian Journal of Mathematics 2 (4), 875-920 (1998).

[31] M. Mulase and P. Sułkowski, Spectral curves and the Schrödinger equations for the Eynard-Orantin recursion, arXiv:1210.3006 [math.ph](2012).

[32] D. Mumford, Projective invariants of projective structures and applications, Proc. Internat. Congr. Mathematicians (Stockholm, 1962), pages 526530. Inst. Mittag-Leffler, Djursholm, 1963.

[33] D. Mumford, Towards an enumerative geometry of the moduli space of curves, in "Arithmetic and Geometry," M. Artin and J. Tate, eds., Part II, 271-328, Birkhäuser, 1983.

[34] D. Mumford, J. Fogarty, and F. Kirwan, Geometric invariant theory, Ergebnisse der Mathematik und ihrer Grenzgebiete, 294 pages, Springer-Verlag (1994).

[35] M.S. Narasimhan and C.S. Seshadri, Stable and unitary vector bundles on a compact Riemann surface, Annals of Mathematics, Second Series, 82, 540-567 (1965).

[36] A. Okounkov and R. Pandharipande, Gromov-Witten theory, Hurwitz numbers, and matrix models, I, Proc. Symposia Pure Math textbf80, 325-414 (2009).

[37] C. S. Seshadri, Space of unitary vector bundles on a compact Riemann surface, Annals of Mathematics, Series $2, \mathbf{8 5}, 303-336$ (1967).

[38] C.T. Simpson, Higgs bundles and local systems, Publications Mathématiques de l'I.H.E.S. 75, 5-95 (1992).

[39] D. D. Sleator, R. E. Tarjan, and W. P. Thurston, Rotation distance, triangulations, and hyperbolic geometry, Journal of the American Mathematical Society 1, 647-681 (1988).

[40] K. Strebel, Quadratic differentials, Springer-Verlag, 1984.

[41] G. 't Hooft, A planer diagram theory for strong interactions, Nuclear Physics B72, 461-473 (1974).

[42] T.R.S. Walsh and A.B. Lehman, Counting rooted maps by genus. I, Journal of Combinatorial Theory B-13, 192-218 (1972).

Olivia Dumitrescu: Pierce Hall 209, Central Michigan University, Mount Pleasant 48859, MichiGAN

and Simion Stoilow Institute of Mathematics, Romanian Academy, 21 Calea Grivitei Street, 010702 Bucharest, Romania

E-mail address: dumit1om@cmich.edu 